ARTICLE

https://doi.org/10.1038/s41467-020-14281-5

\title{
The anterior insular cortex unilaterally controls feeding in response to aversive visceral stimuli in mice
}

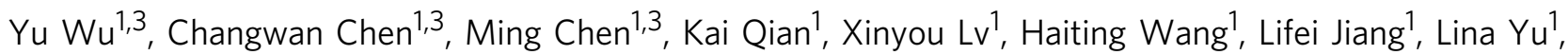
Min Zhuo ${ }^{2} \&$ Shuang Qiu (1) ${ }^{1 \star}$

Reduced food intake is common to many pathological conditions, such as infection and toxin exposure. However, cortical circuits that mediate feeding responses to these threats are less investigated. The anterior insular cortex $(\mathrm{alC})$ is a core region that integrates interoceptive states and emotional awareness and consequently guides behavioral responses. Here, we demonstrate that the right-side alC CamKII+ (alCCamKII) neurons in mice are activated by aversive visceral signals. Hyperactivation of the right-side alCCamKII neurons attenuates food consumption, while inhibition of these neurons increases feeding and reverses aversive stimuli-induced anorexia and weight loss. Similar manipulation at the left-side alC does not cause significant behavioral changes. Furthermore, virus tracing reveals that alCCamKII neurons project directly to the vGluT2 ${ }^{+}$neurons in the lateral hypothalamus $(\mathrm{LH})$, and the rightside alCCamKII-to-LH pathway mediates feeding suppression. Our studies uncover a circuit from the cortex to the hypothalamus that senses aversive visceral signals and controls feeding behavior.

\footnotetext{
${ }^{1}$ Center for Neuroscience and Department of Anesthesiology of Second Affiliated Hospital, NHC and CAMS Key Laboratory of Medical Neurobiology, Zhejiang University School of Medicine, 310058 Hangzhou, Zhejiang, China. ${ }^{2}$ Department of Physiology, Faculty of Medicine, University of Toronto, 1 King's College Circle, Toronto, ON M5S 1A8, Canada. ${ }^{3}$ These authors contributed equally: Yu Wu, Changwan Chen, Ming Chen. *email: qiushly@zju.edu.cn
} 
W hether to eat and how much to eat in any one meal are critical decisions for the health of all metazoan organisms. Under normal conditions, food intake and energy expenditure are balanced by well-defined hypothalamic neural circuits that maintain stable body weight $t^{1-5}$. However, food intake can be attenuated in pathological conditions, such as food poisoning, inflammation, or chemotherapy treatment that may elicit visceral malaise, indicating that some emergencyresponse circuits hijack the energy homeostasis system. However, the neural circuits involved in mediating feeding responses under these non-homeostatic conditions are comparatively elusive.

Insular cortex, especially the anterior part of the insular cortex (aIC), is a critical region for processing salient stimuli and orchestrating appropriate behavioral responses. The aIC receives heavy sensory inputs from the thalamus and serves as the primary visceral and gustatory cortex ${ }^{6-14}$. Neuroimaging studies in humans have revealed that the aIC responds not only to food cues, but also to multiple noxious stimuli that trigger pain, anxiety, and disgust ${ }^{15}$. Studies in patients with eating disorders, such as bulimia nervosa ${ }^{16,17}$ and anorexia nervosa ${ }^{18-20}$, have shown altered insula activation. Animal studies confirmed the activation of the insular cortex in response to anorexigenic signals in vivo ${ }^{21-24}$. Moreover, direct insular stimulation in humans or rodents induces visceral sensations such as nausea 25,26 , whereas damage to the insular cortex blunts lithium-induced malaise ${ }^{21}$ and blocks the behavioral expression of a conditioned taste aversion ${ }^{27-29}$. Although the role of the insular cortex in perceiving multiple aversive signals has been extensively studied, the behavioral responses under these pathological conditions, especially feeding behaviors, upon insular activation are not well studied. By now, a direct role of the insular cortex in controlling food intake has not yet been demonstrated in patients with insular damage $\mathrm{e}^{30}$ or in rodents with insular lesion or inactivation $^{21,31}$

In this study, we demonstrate that the CamKII-positive neurons in the caudal segment of the right-side, but not the left-side, aIC respond to aversive visceral stimuli and suppress feeding via projections to the vGluT2-positive neurons in the lateral hypothalamus (LH). Thus, we identify a role of the aIC that conveys aversive visceral information to the $\mathrm{LH}$ to regulate food consumption.

\section{Results}

The right-side aIC is activated by aversive visceral stimuli. To investigate the involvement of the aIC in processing aversive signals, we monitored Fos expression after intraperitoneal (i.p.) injection of anorexigenic signals, such as lithium chloride ( $\mathrm{LiCl})$, which induces nausea and visceral malaise, lipopolysaccharide (LPS), which triggers a wide range of inflammatory and sickness responses, and cis-diaminodichloroplatinum (Cisplatin) ${ }^{32}$, which is a chemotherapy medication used to treat cancers but induces anorexia and cancer anorexia-cachexia. Immunostaining for Fos revealed that injection (i.p.) of $\mathrm{LiCl}$, LPS, or Cisplatin-induced substantial Fos expression bilaterally in several brain regions, including parabrachial nucleus (PBN), nucleus of solitary tract (NTS), basolateral amygdaloid nucleus (BLA), medial prefrontal cortex (mPFC), LH, the parvicellular part of the ventroposteromedial nucleus of the thalamus (VPMpc), and central amygdala (CeA) (Two-way ANOVA, PBN, $F_{2,15}=0.3023, P=0.0681$; NTS, $F_{2,15}=0.1253, P=0.8831$; BLA, $F_{2,15}=0.562, P=0.5816$; mPFC, $F_{2,15}=0.1878, \quad P=0.8307, \quad L H, \quad F_{2,15}=0.7986, \quad P=0.4682$; VPMpc, $F_{2,15}=0.1315, P=0.8778 ;$ CeA $, F_{2,15}=0.1166, P=$ 0.9493, Supplementary Fig. 1a, b). Of interest, LiCl, LPS, and Cisplatin produced a robust induction of Fos in the right, but not in the left aIC (Two-tailed unpaired $t$ test, Saline, $t_{10}=0.6184$, $P=0.5501 ; \mathrm{LiCl}, t_{22}=4.027, P=0.0006 ; \quad$ LPS, $t_{18}=6.946$,
$P<0.0001$; Cisplatin, $t_{20}=2.886, P=0.0091$, Fig. 1a, b). Further analyses revealed that LiCl-induced or LPS-induced Fos-positive $\left(\right.$ Fos $\left.^{+}\right)$neurons were mainly localized within the caudal segment between $1.26 \mathrm{~mm}$ before Bregma (Bregma +1.26 ) and $0.02 \mathrm{~mm}$ after Bregma (Bregma -0.02) of the right aIC (Two-way ANOVA, Saline, $F_{4,25}=1.961, P=0.1315 ; \mathrm{LiCl}, F_{4,25}=33.42$, $P<0.0001 ; \quad$ LPS $, \quad F_{4,25}=6.957, \quad P=0.00061$, Supplementary Fig. $2 \mathrm{a}-\mathrm{d}$ ). In contrast, injection (i.p.) of cholecysokinin (CCK), a potential satiation factor, or ghrelin, a hunger hormone, resulted in a much less Fos expression (Two-tailed unpaired $t$ test, Ghrelin, $t_{10}=1.607, P=0.1391$; CCK, $t_{10}=0.4837, P=0.639$, Fig. 1a, b) and these $\mathrm{Fos}^{+}$neurons were diffusely distributed within both sides of the aIC (Two-way ANOVA, Ghrelin, $F_{4,25}=$ $1.641, P=0.1612$; CCK, $F_{4,25}=0.6909, P=0.6052$, Supplementary Fig. 2e, f). Additionally, LiCl-induced and LPS-induced Fos ${ }^{+}$ neurons were exclusively located in the agranular part of the caudal segment of the right aIC, whereas CCK-induced Fos ${ }^{+}$ neurons were mainly located in the granular part and Ghrelininduced Fos $^{+}$neurons were diffusely distributed in this segment (one-way ANOVA, Saline, $F_{3,20}=0.6521, P=0.5909$; LiCl, $F_{3,20}=3.416, P=0.0372$; LPS, $F_{3,20}=0.3912, P=0.0212$ Ghrelin, $F_{3,20}=0.6696, P=0.5806 ;$ CCK, $F_{3,20}=2.617, P=$ 0.0477, Supplementary Fig. 2g, h).

Moreover, no obvious Fos expression was observed in the left or right aIC in ad libitum-feeding mice, in 24-h fasted mice or in refeeding mice (two-tailed unpaired $t$ test, home caged, $t_{10}=$ $0.4953, P=0.6311$; fasted, $t_{10}=0.9209, P=0.3788$, re-fed, $t_{10}=$ $0.5575, P=0.5895$, Fig. $1 \mathrm{c}, \mathrm{d})$. We also investigated the mice with electric shock, which exerts no direct effect on the gastrointestinal tract, and observed significant Fos staining in the BLA bilaterally, but not in the aIC (two-way ANOVA, aIC, $F_{1,20}=0.8639, P=$ 0.3637 ; BLA, $F_{1,20}=0.0216, P=0.8846$, Supplementary Fig. 3a, b). Additionally, the right aIC was significantly activated no matter whether $\mathrm{LiCl}$ was injected (i.p.) in the left side or right side of the mouse body, suggesting that the unilateral activation of the aIC is not determined by injection site (two-way ANOVA, left side (i.p.), $F_{1,10}=12.93, P=0.0049$; right side (i.p.), $F_{1,10}=47.22$, $P<0.0001$, Supplementary Fig. 3c). These results indicate that aIC, especially the caudal segment of the right aIC, responds to multiple aversive visceral stimuli.

Notably, double immunohistochemical staining revealed that almost $82.6 \%$ of $\mathrm{Fos}^{+}$neurons activated by $\mathrm{LiCl}$ in the right aIC expressed CamKII, while only $4.1 \%$ of $\mathrm{Fos}^{+}$neurons expressed GAD67 (Fig. 1e, f). To confirm this result, we injected a recombinant adreno-associated virus (AAV) that expressed Camk2a promoter-controlled mCherry (AAV9-CamKIIamCherry) into the caudal segment of the right aIC of the wildtype mice. Three weeks later, we observed that $87.8 \%$ of Fos ${ }^{+}$ neurons activated by $\mathrm{LiCl}$ were colocalized with mCherry-positive cells in this site (Supplementary Fig. 3d). Together, these results indicate that most of the activated neurons are excitatory neurons expressing CamKII.

To determine the dynamics of the CamKII-positive neurons in the caudal segment of the right or left aIC (the right or left aIC CamKII neurons) in vivo during exposure to an aversive stimulus, we performed fiber photometry recording (Fig. 1g, h, Supplementary Fig. 4a). AAV containing GCaMP6f (AAV9mCamKIIa-GCaMP6f) was injected unilaterally into the caudal segment of the right aIC or the left aIC of wild-type mice (Fig. 1g, h). Strikingly, i.p. injection of $\mathrm{LiCl}$ (One-way ANOVA, $F_{3,12}=$ 8.409, $P=0.0028$, Fig. 1i, Supplementary Fig. 4b, c) or Cisplatin (One-way ANOVA, $F_{3,12}=71.38, P<0.0001$, Fig. $1 \mathrm{j}$, Supplementary Fig. $4 \mathrm{~d}, \mathrm{e})$ evoked sustained increase in the activity of the right aIC CamKII neuron, but not in the left aIC CamKII neurons, confirming that aversive visceral stimuli enhance the excitatory neuronal activity in the right aIC. 
a
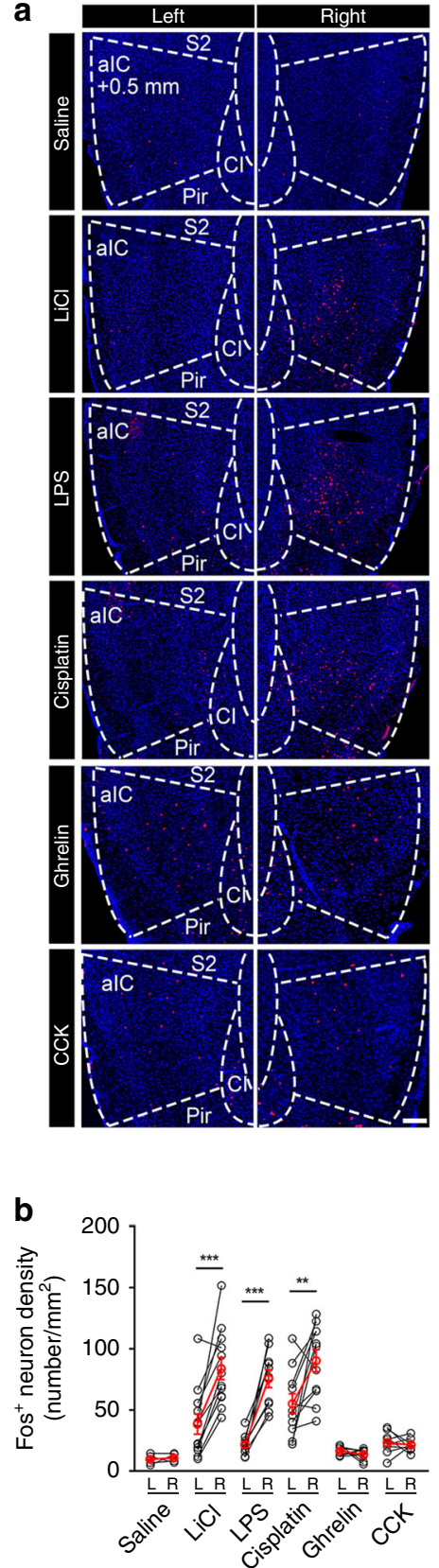

C

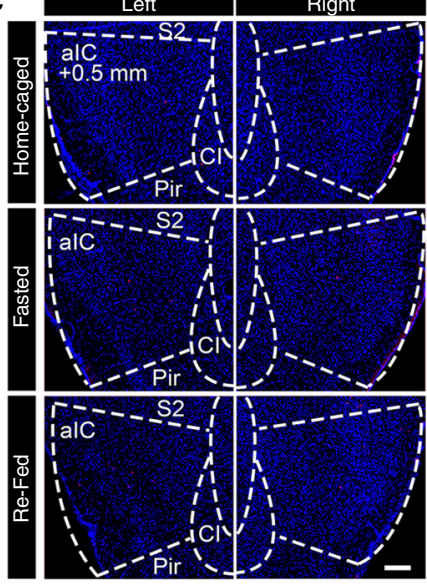

d

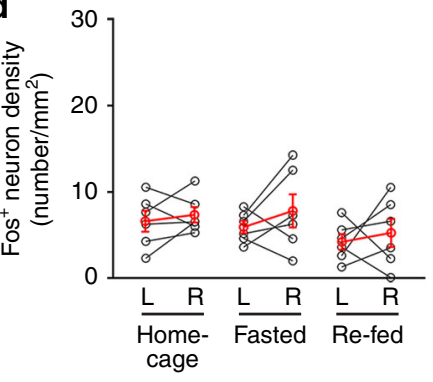

g

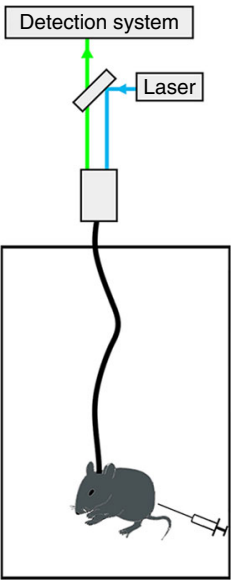

h

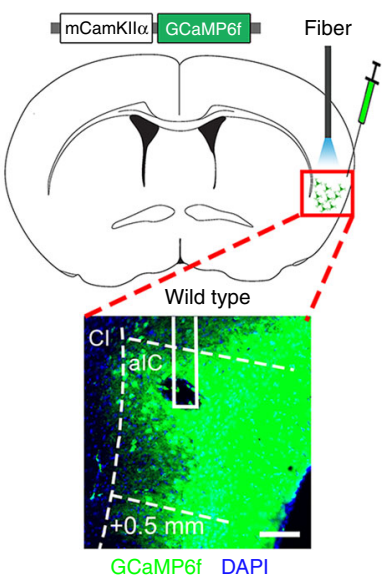

e

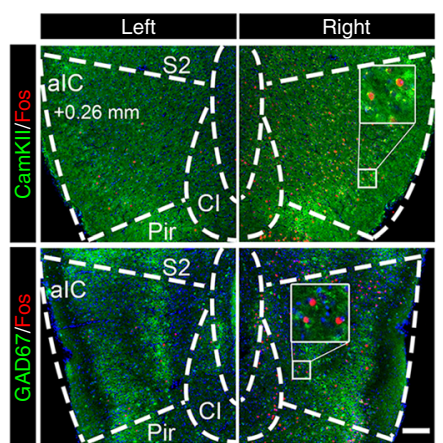

f

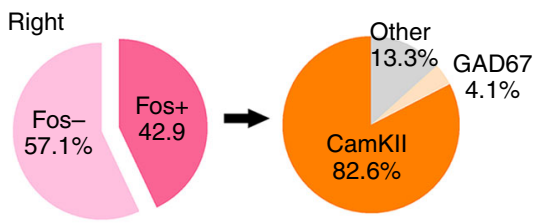

Left

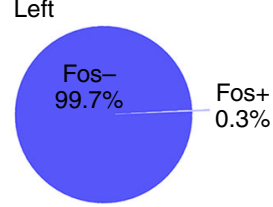

i

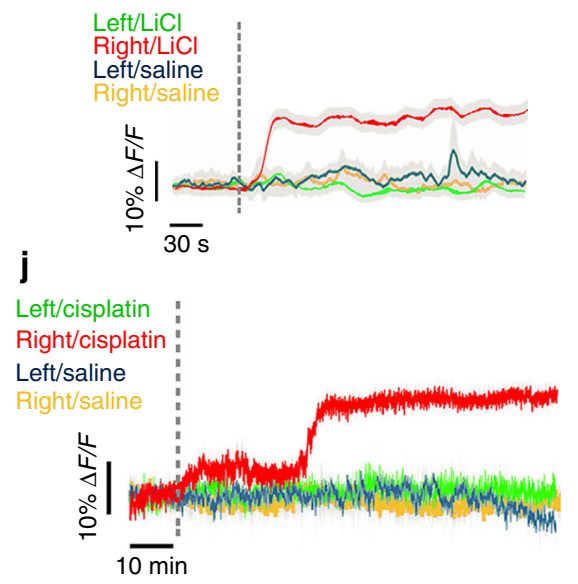

Fig. 1 CamKII ${ }^{+}$neurons in the right alC are activated by aversive visceral stimuli. $\mathbf{a}$, $\mathbf{b}$ Representative histology $\mathbf{a}$ and quantification $\mathbf{b}$ of Fos-like

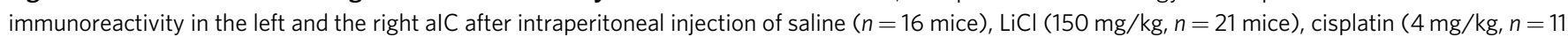
mice), LPS ( $0.1 \mathrm{mg} / \mathrm{kg}, n=14$ mice), Ghrelin ( $1 \mathrm{mg} / \mathrm{kg}, n=6$ mice), or CCK ( $5 \mu \mathrm{g} / \mathrm{kg}, n=6$ mice). Two-tailed unpaired $t$ test, scale bar, $250 \mu \mathrm{m}$. c, d Representative histology $\mathbf{c}$ and quantification $\mathbf{d}$ of Fos-like immunoreactivity in the left and the right alC in fed or 24-h fasted mice with or without 3-h refeeding ( $n=6$ mice per group. Two-tailed unpaired $t$ test). Scale bar, $250 \mu \mathrm{m}$. e, f Co-expression e of CamKII or GAD67 (green) with Fos (red) in the right or left alC injection with $\mathrm{LiCl}$ and quantification $\mathbf{f}$. $n=6$ mice per group. Scale bar, $100 \mu \mathrm{m}$. $\mathbf{g}$ Schema of the fiber photometry approach. $\mathbf{h}$ Image of GCaMP6f expression in the right alCCamKII, with the fiber track above. Scale bar, $500 \mu \mathrm{m}$. $\mathbf{i}$, j Representative mean GCaMP6f fluorescence responses of both sides of the alC CamKII neurons by injection of Saline, LiCl, or Cisplatin. i.p. intraperitoneal. $n=4$ mice per group. For $\mathbf{b}$ and $\mathbf{d}$, red line represents averaged data. ${ }^{\star \star} P<0.01 ;{ }^{\star \star \star} P<0.005$. Data are presented as means \pm SEM. Source data are provided as a Source Data file.

Activating the right-side aIC CamKII neurons impairs feeding. To explore whether the $\mathrm{CamKII}^{+}$neurons in the caudal segment of the right aIC mediate feeding behavior, we injected Credependent AAV containing a double floxed inverted orientation (DIO) excitatory optogenetic receptor construct (AAV8-DIOhChR2(H134R)-eGFP) into the caudal segment of the right aIC of Camk2a-Cre transgenic mice (Fig. 2a, Supplementary Fig. 5a). Whole-cell patch-clamp recordings in the acute brain slices showed that action potentials of ChR2-expressing aIC CamKII neurons were triggered by 473-nm light pulses (Fig. 2b). We next performed a time-resolved analysis of feeding in mice that were deprived of food for $24 \mathrm{~h}$ (Fig. 2c). Photoactivation of the right aIC CamKII neurons at $20 \mathrm{~Hz}$ significantly decreased the amount of food intake (two-tailed unpaired $t$ test, $t_{17}=2.385, P=0.029$ ) and the percentage of time spent feeding (feeding time was determined as the time the mice successfully accomplished 
a
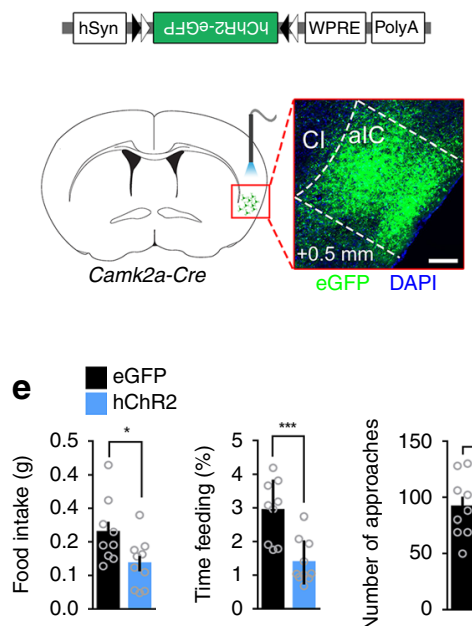

i

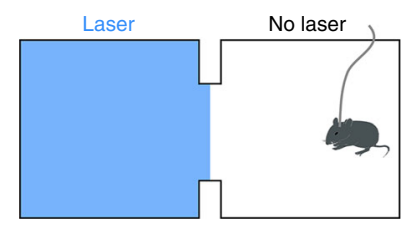

b

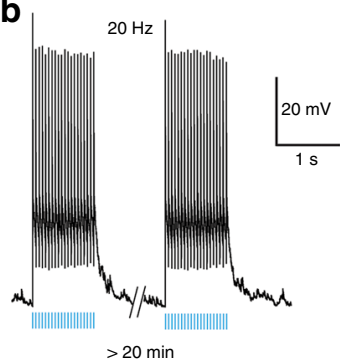

f

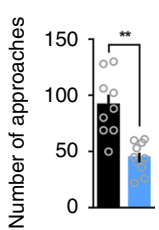

C
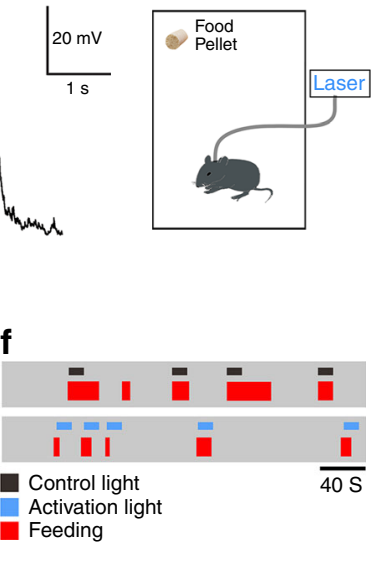

d

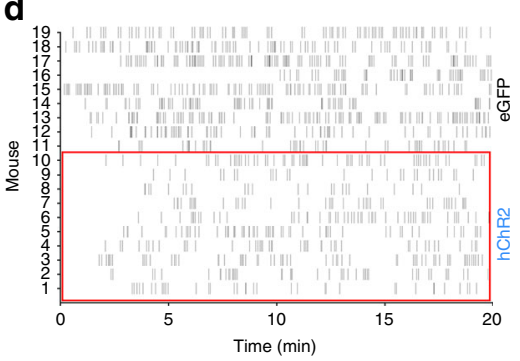

g

h

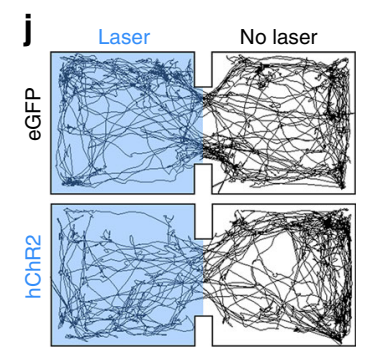

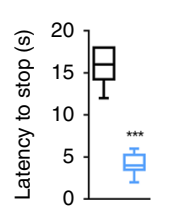

$\mathbf{k}$
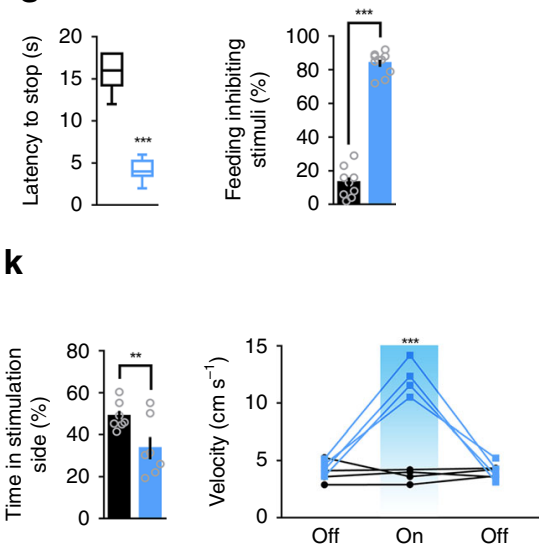

Fig. 2 Activation of the right alCCamKII neurons suppresses food intake. Diagrams illustrating the injected virus (upper) and injection site in the caudal segment of the right alC (lower). The inserted image shows the expression of hChR2 (H134R)-eGFP in the right alC. WPRE woodchuck hepatitis virus posttranscriptional regulatory element; pA poly $\mathbf{a}$; $\mathrm{Cl}$ claustrum. Scale bar, $500 \mu \mathrm{m}$. b Brain slice whole-cell patch-clamp recording traces showing action potentials triggered in the alCCamKII neurons expressing hChR2 by $20 \mathrm{~Hz}, 10 \mathrm{~ms}, 473 \mathrm{~nm}$ laser pulses. c Schema to assess food intake for optogenetics. d Raster plot of food approaches in 24-h fasted mice expressing hChR2(H134R)-eGFP in the right alC. e Total food consumption, percentage of time spent feeding, number of approaches to food and latency of the first approach to food were measured during a 20-min test $(n=9$ for eGFP mice, $n=10$ for hChR2 mice, Two-tailed unpaired $t$ test). f Raster plots showing feeding processes by one 24-h fasted mouse in its home cage. Activation light (473 nm) or control light $(589 \mathrm{~nm})$ was triggered after feeding began. $\mathbf{g}$ Latency to stop feeding in response to light activation ( $473 \mathrm{~nm}), 30 \mathrm{trails}$ from five animals ( $n=5$ mice per group, Two-tailed unpaired $t$ test). $\mathbf{h}$ The percentage of light stimuli $(473 \mathrm{~nm})$ that inhibits feeding $(n=9$ for eGFP mice, $n=10$ for hChR2 mice, Two-tailed unpaired $t$ test). i Schema depicting the real-time place performance (RTPP) paradigm. $\mathbf{j}$ Representative locomotor trace of a mouse with the caudal segment of the right alC CamKII expressing eGFP or hChR2 that received the $20-\mathrm{Hz}$ photostimulation in the laser compartment. $\mathbf{k}$ Percentage of time spent (left) and locomotor activity (right) in laser or no laser side ( $n=9$ for eGFP mice; $n=10$ for hChR2 mice, Two-tailed unpaired $t$ test). ${ }^{\star} P<0.05$; ${ }^{\star \star} P<0.01 ;{ }^{\star \star \star} P<0.005$. Data are presented as means \pm SEM. Source data are provided as a Source Data file.

touching and consuming a food pellet, Two-tailed unpaired $t$ test, $t_{17}=4.259, P=0.005$ ) during a 20 -min test (Fig. 2d, e). Moreover, photoactivation of these neurons decreased the number of approaches (the number of times at which the mice's nose touched the food pellet, two-tailed unpaired $t$ test, $t_{17}=4.775, P=$ $0.0067)$ and increased the latency of the first approach to food (two-tailed unpaired $t$ test, $t_{17}=3.408, P=0.0034$ ) (Fig. $2 \mathrm{~d}$, e). Activation of these neurons also interrupted ongoing feeding within a few seconds following the onset of photoactivation in 24$\mathrm{h}$ fasted mice when mice were in their home cages (Fig. $2 \mathrm{f}-\mathrm{h}$, Two-tailed unpaired $t$ test, for latency to stop, $t_{20}=10.7, P<$ 0.0001; for the percentage of stimuli that inhibits feeding, $t_{17}=$ 19.64, $P<0.0001)$. Thus, activation of the CamKII ${ }^{+}$neurons in the caudal segment of the right aIC inhibited both the appetitive and consummatory phases of feeding behaviors.

We further tested whether activation of these neurons affects the valence of taste, cardiac function, or other classical consummatory behaviors and observed that optogenetic activation of these neurons has no effect on the mice bitter sensitivity (two-way ANOVA, $F_{3,56}=0.6245, P=0.6022$, Supplementary Fig. 5b), heart rate (Two-tailed unpaired $t$ test, $t_{6}=0.9329, P=$ 0.3869 , Supplementary Fig. $5 \mathrm{c}$, d), drinking (two-tailed unpaired $t$ test, Water intake, $t_{12}=0.1997, P=0.8457$; Number of licks, $t_{12}=0.2915, \quad P=0.7756 ;$ Time drinking, $t_{12}=0.1993, \quad P=$ 0.8454, Supplementary Fig. 5e, f) or mating behaviors (twotailed unpaired $t$ test, $t_{68}=0.9092, P=0.3702$, Supplementary Fig. 5g-i).

In the following, we tested the approach or avoidance behavior using real-time place preference assay (RTPP) and observed that mice expressing hChR2 in right aIC CamKII neurons spent significantly less time on the photostimulated side and made more escape attempts (two-tailed unpaired $t$ test, Time in stimulation side, $t_{12}=2.915, P=0.0095$; Velocity, 1st light off step, $t_{17}=0.754, P=0.4772$, 1st light on step, $t_{17}=10.23, P<$ $0.0001,2$ nd light off step, $t_{17}=0.262, P=0.802$, Fig. $2 \mathrm{i}-\mathrm{k}$ ). In contrast, optogenetic activation of these neurons induced no anxiety-like behaviors in two different assays of anxiety, including the elevated plus maze test (two-tailed unpaired $t$ test, Open arm entries, $t_{14}=0.7477, P=0.467$; Time in open arm, $t_{14}=0.3227$, $P=0.7517$, Supplementary Fig. 5j, k) and open field test (OFT) (two-tailed unpaired $t$ test, time in center, $t_{15}=0.3872$, $P=0.7041$; velocity, $t_{15}=0.777, \quad P=0.4492$, Supplementary Fig. 5l, m).

To confirm the specificity of the caudal segment of the right aIC in mediating feeding behavior, we optogenetically activated the $\mathrm{CamKII}^{+}$neurons in the rostral segment of the right aIC 

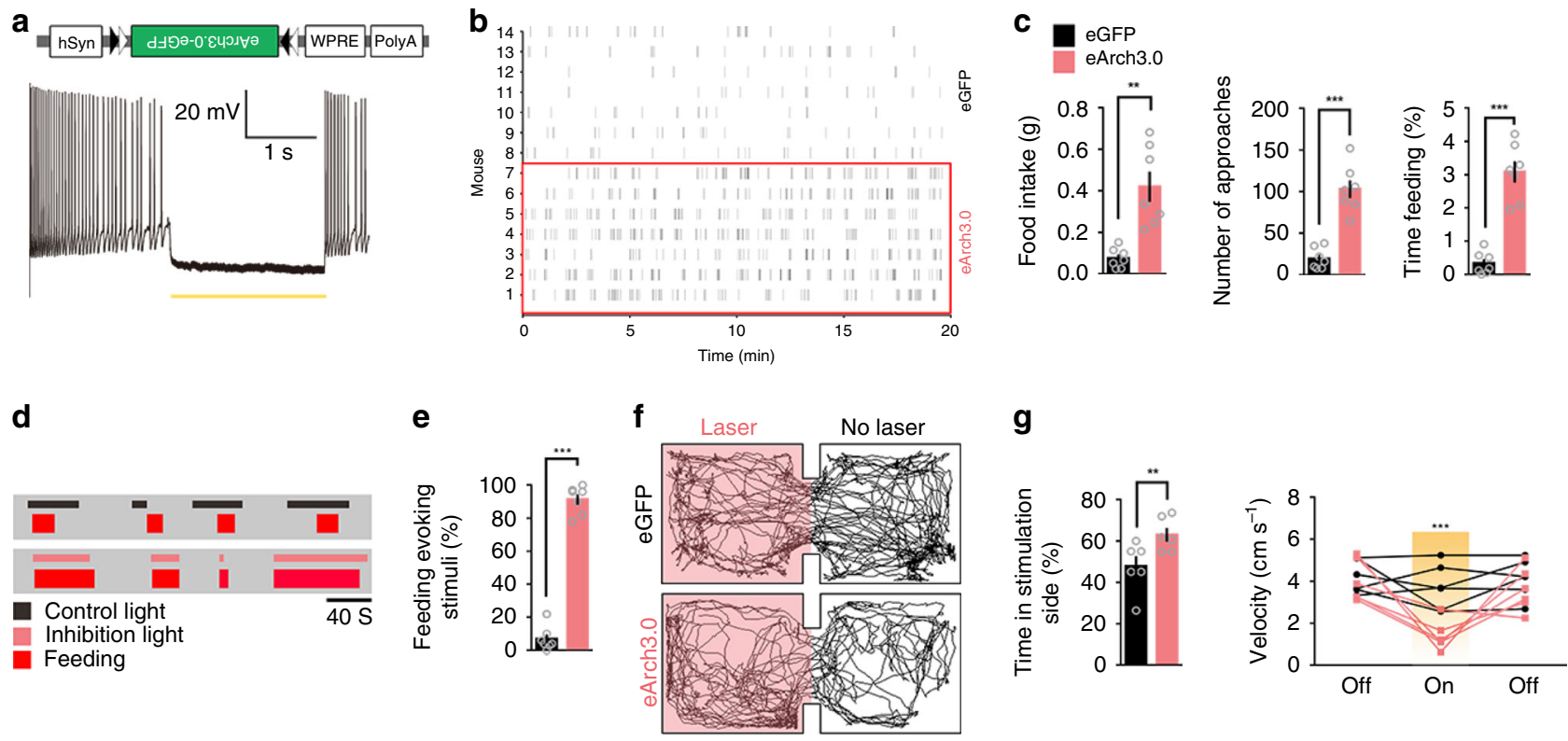

Fig. 3 Inhibition of the right alCCamKII neurons promotes food intake. a Diagrams illustrating the injected virus (upper) and sample slice recordings from the alCCamKII neurons expressing eArch3.0 (orange lines, 593-nm light) (lower). b Raster plot of feeding bouts in the fed mice expressing eArch3.0-eGFP in the caudal segment of the right alC. c Total food consumption (left), number of approaches to food (middle) and the percentage of feeding time (right) were measured during a 20-min test ( $n=7$ mice for each group, Two-tailed unpaired $t$ test). $\mathbf{d}$ Raster plots showing feeding processes by one fed mouse in its home cage. Inhibition light $(532 \mathrm{~nm})$ and control light $(644 \mathrm{~nm})$ was triggered randomly. $\mathbf{e}$ The percentage of light stimuli (532 nm) that induces feeding ( $n=7$ mice for each group, Two-tailed unpaired $t$ test). $\mathbf{f}$ Representative locomotor trace of a mouse with the caudal segment of the right alC CamKII neurons expressing eGFP or eArch3.0 that received photoinhibition in the laser compartment. $\mathbf{g}$ Percentage of time spent (left) and locomotor activity (right) in laser or no laser sides ( $n=7$ mice per group, Two-tailed unpaired $t$ test). ${ }^{\star} P<0.05$; ${ }^{\star \star} P<0.01 ;{ }^{\star \star \star} P<0.005$. Data are presented as means \pm SEM. Source data are provided as a Source Data file.

(two-tailed unpaired $t$ test, $t_{12}=0.569, P=0.5799$, Supplementary Fig. $6 \mathrm{a}, \mathrm{b}$ ) or those in the right posterior IC (pIC) (two-tailed unpaired $t$ test, $t_{13}=0.5801, P=0.5718$, Supplementary Fig. 6c-e) and observed that photoactivation of the CamKII ${ }^{+}$neurons in these two neighboring sites has no effect on feeding behavior.

Silencing the right-side aIC CamKII neurons promotes feeding. We next asked whether inhibition of the CamKII ${ }^{+}$neurons in the caudal segment of the right aIC increases food consumption under conditions in which motivation to eat is low. We silenced the right aICCamKII neurons expressing Cre-dependent AAV9DIO-eArch3.0-eGFP virus with 532-nm laser light (Fig. 3a upper, Supplementary Fig. 7a). Brain slice recordings confirmed that aICCamKII neurons expressing eArch3.0 could be strongly inhibited by $532-\mathrm{nm}$ laser light (Fig. 3a lower). Indeed, optogenetic silencing of the $\mathrm{CamKII}^{+}$neurons in the caudal segment of the right aIC increased the total amount of food intake (Two-tailed unpaired $t$ test, $t_{12}=3.758, P=0.049$ ), the number of approach (Two-tailed unpaired $t$ test, $t_{12}=7.167, P<0.0001$ ) and the percentage of time spent feeding (Two-tailed unpaired $t$ test, $\left.t_{12}=7.995, P<0.0001\right)$ in fed mice expressing eArch3.0, but not in mice expressing eGFP (Fig. 3b, c). Moreover, silencing these neurons evoked feeding behavior in fed mice when the mice were in their home cages (Two-tailed unpaired $t$ test, $t_{12}=20.14$, $P<0.0001$, Fig. 3d, e). These data suggest that inhibition of neural activity within the caudal segment of right aIC is sufficient to induce feeding behavior.

In the RTPP assay, the mice expressing eArch3.0 in these neurons exhibited a significant preference for the photoinhibitionpaired chamber (Fig. $3 \mathrm{f}-\mathrm{g}$, Two-tailed unpaired $t$ test, Time in stimulation side, $t_{12}=3.915, P=0.0083$; Velocity, 1st light off step, $t_{12}=0.3843, P=0.7097$, 1st light on step, $t_{12}=0.676$, $P=0.515$, 2nd light off step, $\left.t_{12}=0.6583, P=0.5268\right)$. In contrast, drinking (two-tailed unpaired $t$ test, $t$ test, water intake, $t_{12}=1.96, P=0.0737$; number of licks, $t_{12}=1.503, P=0.1587$; time drinking, $t_{12}=0.1523, P=0.1523$, Supplementary Fig. $7 \mathrm{~b}$ ), mating (two-tailed unpaired $t$ test, $t$ test, $t_{34}=1.335, P=0.1908$, Supplementary Fig. 7c), and anxiety-like behaviors (Supplementary Fig. 7d-g, Two-tailed unpaired $t$ test, for Supplementary Fig. 7e, open arm entries, $t_{12}=0.6325, P=0.539$; time in open arm, $t_{12}=0.874, P=0.3993$, for Supplementary Fig. $7 \mathrm{~g}$, time in center, $t_{12}=1.037, P=0.3202$; velocity, $t_{12}=1.078, P=0.3022$ ) were unaffected. Taken together, these results indicate that the inhibition of the CamKII ${ }^{+}$neurons in the caudal segment of the right aIC promotes food consumption in the absence of homeostatic deficit and at the same time induces approach behavior.

Activating the left aIC CamKII neurons induces no feeding. We also explored whether the caudal segment of the left aIC CamKII neuron activity is involved in mediating feeding or avoidance behavior. AAV virus carrying hChR2 was injected unilaterally in the caudal segment of the left aIC of Camk2a-Cre mice (Fig. 4a, Supplementary Fig. 8a). Optogenetic activation of these neurons in vivo at $20 \mathrm{~Hz}$ had no effect on feeding behavior in 24-h-fasted mice (two-tailed unpaired $t$ test, food intake, $t_{12}=0.3688$, $P=0.7187$; number of approaches, $t_{12}=0.9881, P=0.3426$; time feeding, $t_{12}=0.2507, P=0.8063$, Fig. $\left.4 \mathrm{~b}, \mathrm{c}\right)$. Moreover, activation of these neurons produced no conditioned place aversion (Twotailed unpaired $t$ test, time in stimulation side, $t_{12}=0.4276$, $P=0.6765$; velocity, 1 st light off step, $t_{12}=0.493, P=0.631,1$ st light on step, $t_{12}=0.8914, P=0.3902$, 2nd light off step, $t_{12}=0.5257, P=0.6087$, Fig. $4 \mathrm{~d}, \mathrm{e}$ ), induced no drinking (Twotailed unpaired $t$ test, water intake, $t_{12}=0.1104, P=0.9139$; number of licks, $t_{12}=0.5958, P=0.5624$; time drinking, $t_{12}=0.3115, P=0.7607$, Supplementary Fig. 8b) or mating 
a

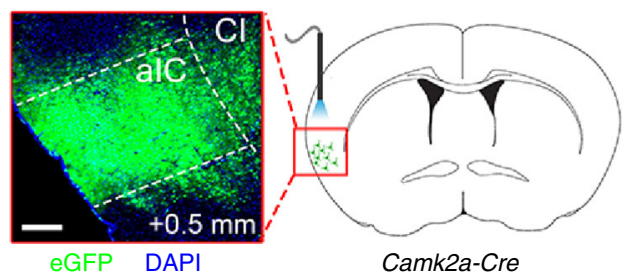

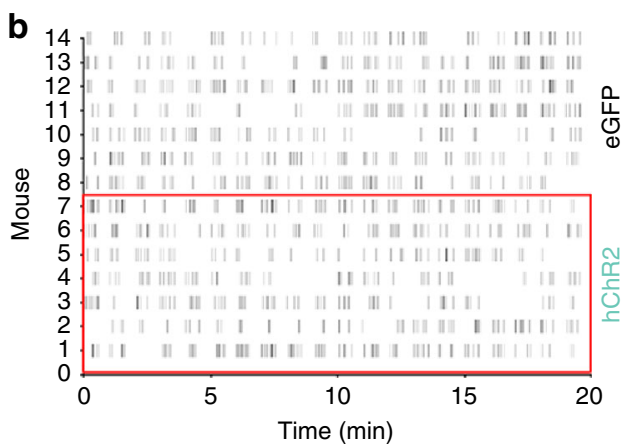

C

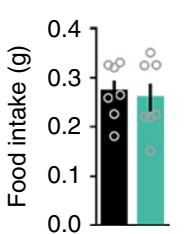

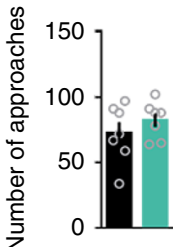

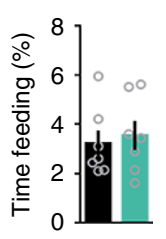

d

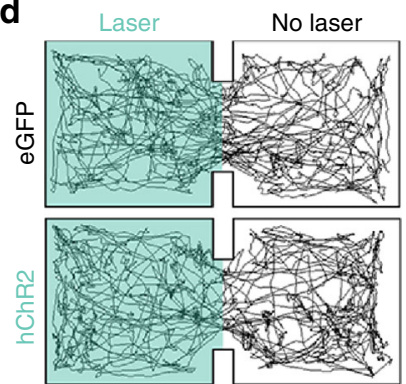

e
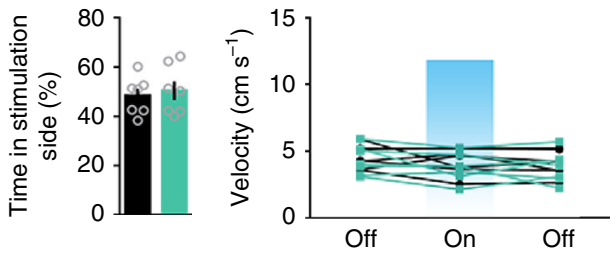

Fig. 4 Activation of the left aICCamKII neuron has no effect on feeding behavior. a The inserted image shows the expression of hChR2-eGFP in the caudal segment of the left alC. Scale bar, $250 \mu \mathrm{m}$. b Raster plot of approaches to food in 24-h fasted mice expressing hChR2-eGFP in the caudal segment of the left alC and receiving $20 \mathrm{~Hz}$ photostimulation. c Total food consumption (left), number of approaches (middle) and the percentage of feeding time (right) were measured ( $n=7$ mice per group, Two-tailed unpaired $t$ test). d Representative locomotor trace of a mouse with the caudal segment of the left alC CamkII neurons expressing eGFP or hChR2 and receiving $20 \mathrm{~Hz}$ photostimulation in the laser compartment. e Percentage of time spent in the photostimulation side (left) and locomotor activity (right) in laser or no laser sides ( $n=7$ mice per group, Two-tailed unpaired $t$ test). Data are presented as means \pm SEM. Source data are provided as a Source Data file.

behavior (two-tailed unpaired $t$ test, $t_{27}=0.8559, P=0.3996$, Supplementary Fig. 8c), and exerted no effect on anxiety-like behavior (Supplementary Fig. $8 \mathrm{~d}-\mathrm{g}$; two-tailed unpaired $t$ test, for Supplementary Fig. $8 d, t_{27}=0.8559, P=0.3996$; for Supplementary Fig. $8 \mathrm{e}$, open arm entries, $t_{12}=0.3174, P=0.7564$; time in open arm, $t_{12}=0.1824, P=0.8583$; for Supplementary Fig. $8 \mathrm{~g}$, time in center, $t_{12}=0.0082, P=0.9936$; velocity, $t_{12}=0.0059$, $P=0.9953)$. These data suggest that the caudal segment of the left aIC is unnecessary for feeding or avoidance behavior.

Inhibiting the right aIC CamKII neurons reverses anorexia. To test the long-term effect of the right aICCamKII neuron activity, we injected an excitatory virus AAV9-DIO-hM3Dq-mCherry or inhibitory virus AAV9-DIO-hM4Di-mCherry in the caudal segment of the right aIC of Camk2a-Cre mice (Supplementary Fig. 9a, b). Electrophysiological analysis in acute slices confirmed that Clozapine N-oxide (CNO, $5 \mu \mathrm{M}$ ) activated spiking in neurons expressing hM3Dq (Fig. 5a, b). CNO treatment rapidly and markedly inhibited food intake in $24 \mathrm{~h}$-fasted mice expressing $\mathrm{hM} 3 \mathrm{Dq}$ in the caudal segment of the right aIC CamKII neurons during a 1 -h test (Two-way ANOVA, food intake, $F_{1,18}=43.11$, $P<0.0001$; feeding time, $F_{1,18}=32.82, P<0.0001$, Fig. $5 c$ ). Moreover, long-term $\mathrm{CNO}$ treatment significantly reduced body weight in mice expressing $\mathrm{hM} 3 \mathrm{Dq}$ in these neurons but not in mice expressing mCherry. Body weight returned to normal after cessation of CNO treatment (two-way RM ANOVA, interaction: $F_{19,114}=10.6, P<0.0001$, Fig. 5d).

Electrophysiological analysis in acute slices also confirmed that CNO $(5 \mu \mathrm{M})$ inhibited spiking in neurons expressing hM4Di (Fig. 5e, f). Of interest, administration of $\mathrm{CNO}$ (i.p.) to inhibit the $\mathrm{CamKII}^{+}$neurons in the caudal segment of the right aIC had no effect on CCK-induced reduction of food intake in fasted mice (two-way ANOVA, saline, $F_{3,26}=1.113, P=0.3619$; CCK,
$F_{3,26}=2.238, \quad P=0.1076$, Fig. $5 \mathrm{~g}$ ). However, chemogenetic inhibition of these neurons prevented LiCl-induced and LPSinduced reduction of food intake (two-way ANOVA, LiCl, $F_{3,26}=3.287, P=0.0365$; LPS, $F_{3,26}=9.614, P=0.0002$, Fig. $5 \mathrm{~g}$ ). Administration of saline (i.p.) has no effect on CCK-induced, LiCl-induced, or LPS-induced reduction of food intake (two-way ANOVA, Saline, $F_{3,26}=0.4549, P=0.7161$; CCK, $F_{3,26}=0.4727$, $P=0.7039 ; \mathrm{LiCl}, F_{3,26}=0.7194, P=0.5394 ; \mathrm{LPS}, F_{3,26}=0.6813$, $P=0.5715$, Supplementary Fig. 9c). Moreover, long-term CNO treatment significantly increased the body weight of mice expressing hM4Di in the $\mathrm{CamKII}^{+}$neurons in the caudal segment of the right aIC, but not mice-expressing mCherry, and this effect was reversed post CNO injection (two-way RM ANOVA, interaction: $F_{18,126}=9.078, P<0.0001$, Bonferroni post hoc analysis mCherry with hM4Di on days $6-8$ as indicated, Fig. 5h). Long-term Cisplatin treatment (every 2 days for 14 days) significantly reduced the body weight of the injected mice. Chronic chemogenetic inhibition of the right aIC CamKII neurons significantly prevented further weight loss, while inhibition of the left aIC CamKII neurons failed to rescue body weight loss (two-way RM ANOVA, interaction: $F_{16,64}=57.1, P<0.0001$, Bonferroni post hoc analysis mCherry with hM4Di on days 9-14 as indicated, Fig. 5i), implying that the caudal segment of the right aIC is critical in mediating feeding in response to aversive stimuli.

The aIC CamKII neurons directly innervate LH $^{\text {vGluT2 }}$ neurons. To elucidate the neurocircuitry involved in the aIC-mediated feeding behavior, we anatomically mapped the long-range projections of $\mathrm{CamKII}^{+}$neurons in the aIC. First, we selectively expressed a Cre-dependent fluorophore AAV8-DIO-eGFP into the right aIC of Camk2a-Cre mice. Imaging showed that the aIC CamKII neurons projected heavily to the hypothalamus (Supplementary Fig. $10 \mathrm{a}, \mathrm{b}$ ). It is of interest to note that, in the 
a

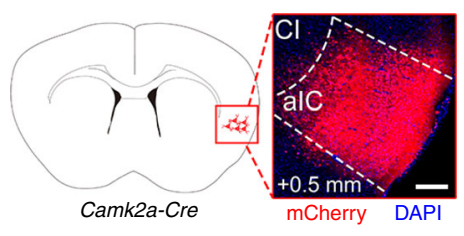

b

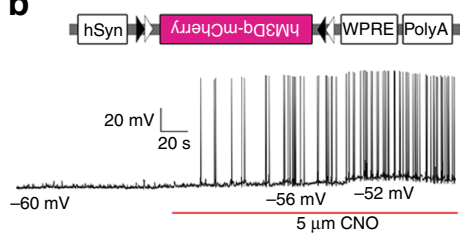

e

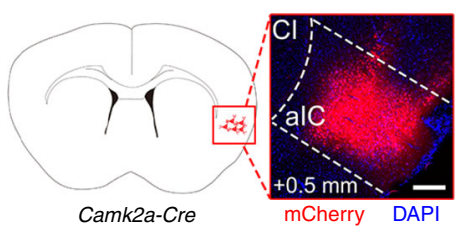

Week d

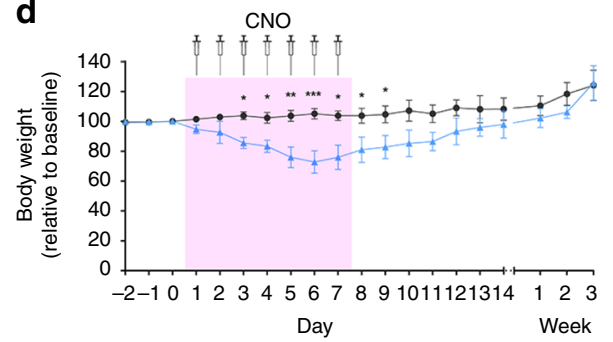

g

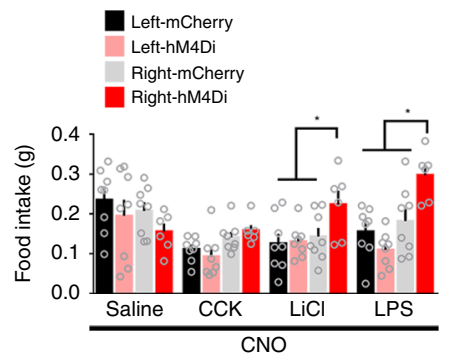

h

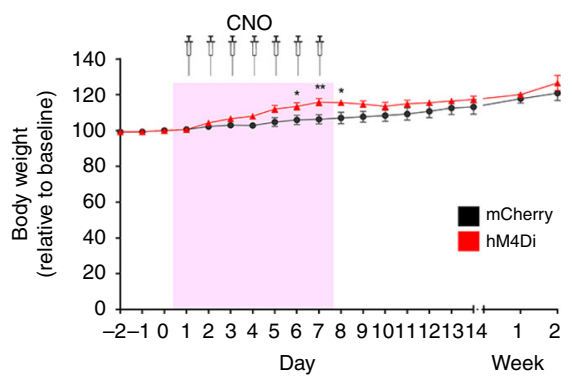

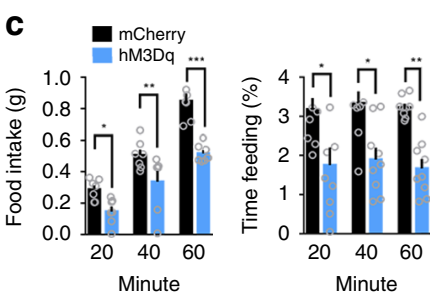

f

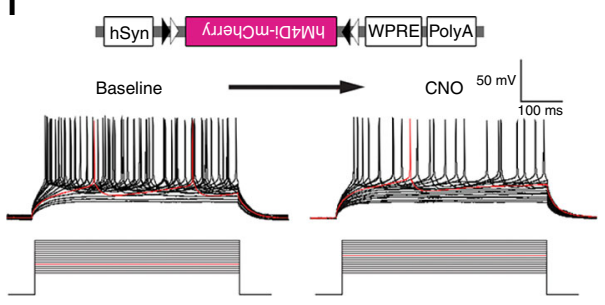

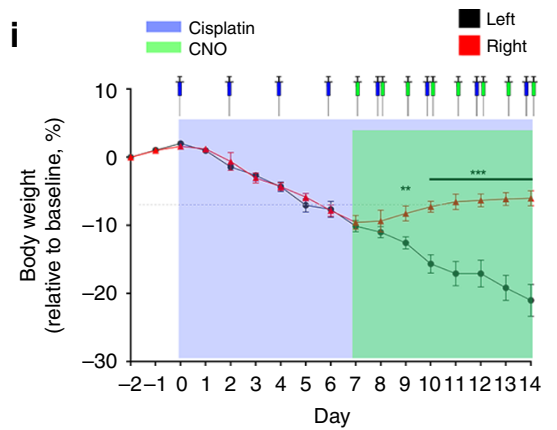

Fig. 5 Inhibition of the right alC CamKII neurons rescues Cisplatin-induced anorexia. a The inserted image shows the expression of hM3Dq-mCherry in the caudal segment of the right alC. Scale bar, $250 \mu \mathrm{m}$. b Diagrams illustrating the injected virus (upper) and example current-clamp traces from the brain slice with the right alCCamKII neurons expressing hM3Dq before and after treatment with CNO. c Food intake (left) and percentage of feeding time (right) in CNO-treated $24 \mathrm{~h}$-fasted mice expressing hM3Dq in the right alCCamKII neurons during 20, 40, or 60-min test ( $n=7$ mice for per group). d Chronic administration of CNO (pink area, $1 \mathrm{mg} / \mathrm{kg}$, every $12 \mathrm{~h}$ for 7 days, i.p.) reduces body weight ( $n=8$ mice per group). e The inserted image shows the expression of hM3Di-mCherry in the caudal segment of the right alC. Scale bar, $250 \mu \mathrm{m}$. $\mathbf{f}$ Diagrams illustrating the injected virus (upper) and current-voltage relationship of a representative alC CamKII neuron recorded before and after perfusion with CNO. Raw traces show individual voltage responses to a series of 500 ms current pulses from 100 to 200 pA with 10-pA steps. Red traces indicate the minimal current to induce action potentials. g Food intake in $24 \mathrm{~h}$-fasted mice after administration of different anorexigenic agents (CCK $(5 \mu \mathrm{g} / \mathrm{kg}), \mathrm{LiCl}(150 \mathrm{mg} / \mathrm{kg}), \mathrm{and} \mathrm{LPS}(0.1 \mathrm{mg} / \mathrm{kg})$ and CNO ( $n=8$ for left-mCherry, left-hM4Di, and right-mCherry group, $n=6$ for right-hM4Di group). $\mathbf{h}$ Chronic administration of CNO (pink area, i.p.) increases body weight ( $n=9$ mice for control group, $n=8$ mice for hM4Di group). i Chronic administration of Cisplatin (blue area, $4 \mathrm{mg} / \mathrm{kg}$, i.p.) every 2 days for 14 days followed by CNO injection (green area, i.p.) every $12 \mathrm{~h}$ for 7 days after 7 days Cisplatin injection. Body weight of mice was measured every day $\left(n=8\right.$ mice per group). Two-way ANOVA with Bonferroni post hoc analysis. ${ }^{\star} P<0.05$; ${ }^{\star \star} P<0.01$; ${ }^{\star \star \star} P<0.005$. Data are presented as means \pm SEM. Source data are provided as a Source Data file.

hypothalamus, fiber terminals originating from the aIC were predominantly localized within the LH (Fig. 6a), a critical neuroanatomical substrate for feeding behavior ${ }^{33}$. Next, we designed a series of experiments to test whether the aIC CamKII neurons have direct projections to the LH. Immunohistochemical staining showed that stimulation of the projection terminals with $473-\mathrm{nm}$ laser induced significant Fos expression in the entire length of the anterior-posterior (A-P) axis of the $\mathrm{LH}$, but a greater number between 1.06 and $1.58 \mathrm{~mm}$ posterior of Bregma (Twoway ANOVA, Fos expression at $-0.7 \mathrm{~mm}, F_{2,28}=1.364, P=$ 0.5503 ; Fos expression at $-1.06 \mathrm{~mm}, F_{2,28}=2.871, P=0.073$; Fos expression at $-1.34 \mathrm{~mm}, F_{2,28}=43.47, P<0.0001$; Fos expression at $-1.58 \mathrm{~mm}, F_{2,28}=5.581, P=0.0031$; Fos expression at $-1.94 \mathrm{~mm}, F_{2,28}=3.554, P=0.041$, Fig. 6b), indicating that terminals originating from the aIC form functional synaptic connections with the neurons in the LH. To characterize the aIC to $\mathrm{LH}$ connection, whole-cell electrophysiological recording was used to examine light-evoked inhibitory postsynaptic currents (IPSCs) and excitatory postsynaptic currents (EPSCs) from LH neurons in brain slices. This was accomplished by alternation between the holding potential of $0 \mathrm{mV}$ (IPSCs) and $-70 \mathrm{mV}$ (EPSCs) (Fig. 6c). Light activation resulted in eEPSCs and no eIPSCs in all cells tested. Moreover, evoked EPSCs were blocked by the glutamatergic receptor antagonist CNQX (Fig. 6d), indicating that the terminals originating from the aIC formed functional glutamatergic synaptic connections with the neurons in the LH. To further test the cell-type specificity of this projection, we performed Cre-dependent, rabies virus-based retrograde tracing from vGluT2 neurons of the LH. We injected synaptic retrograde $\Delta \mathrm{G}$-rabies viruses encoding $\mathrm{mCh}(\mathrm{RV})$ into the $\mathrm{LH}$ of Slc17a6 (encodes vesicular glutamate transporter, vGluT2)-ires-Cre mice. Positive signals were detected in the aIC and colocalized well with CamKII (Fig. 6e, f), which confirms that the aIC CamKII neurons have direct projection to the vGluT2 ${ }^{+}$neurons in the LH.

To confirm that the aIC receives input from thalamus, we further injected retrograde cholera toxin subunit B (CTB, conjugated with Alexa-555) into the right aIC and observed significant signals in the area VPMpc (Supplementary Fig. 10c). 
a
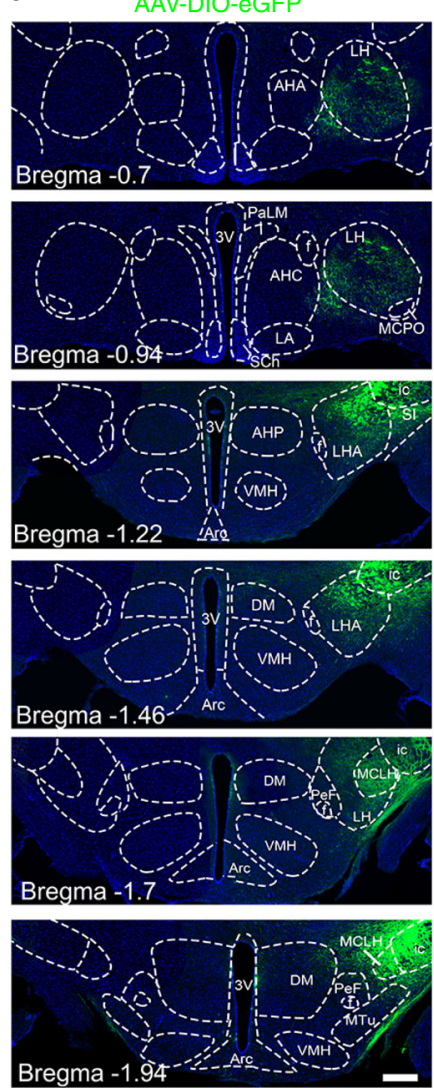

b
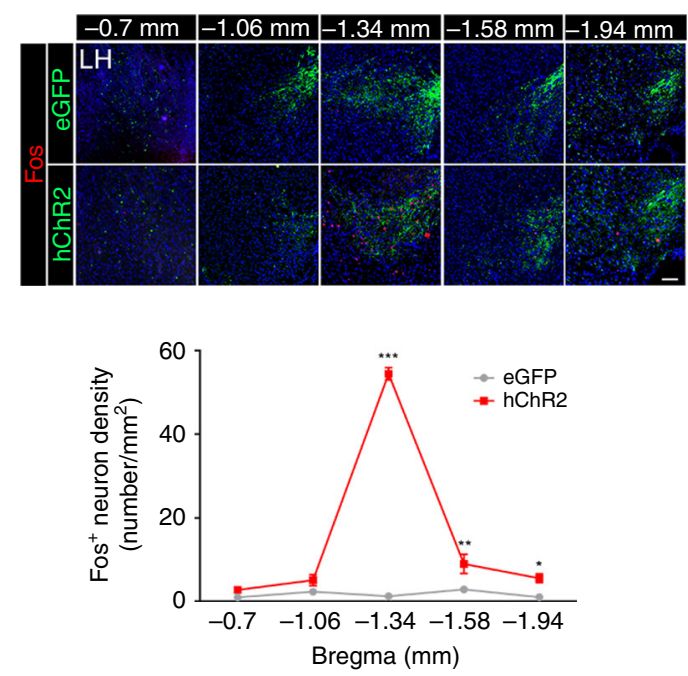

C

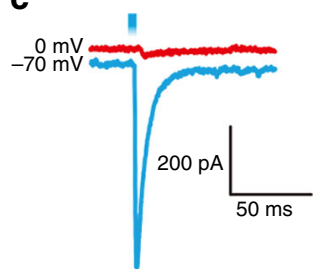

e

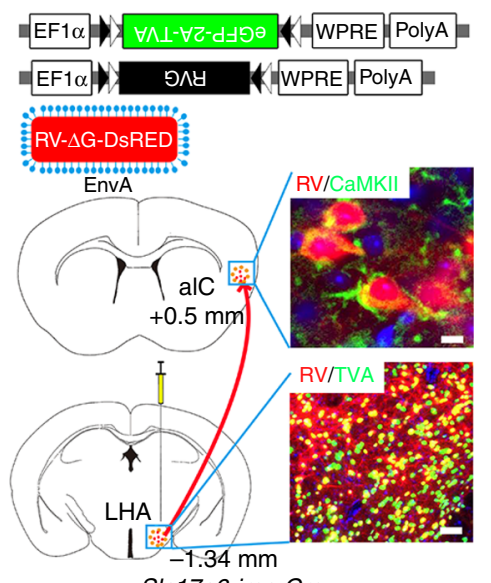

Slc17a6-ires-Cre

$\mathbf{f}$

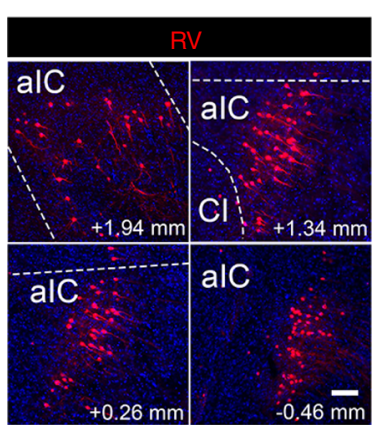

Fig. 6 Right alCCamKII neurons project to the LH directly. a Representative histology of images from the LH showing CamKII+ fiber terminal in green after injecting hSyn-DIO-eGFP into the right aIC of Camk2a-Cre mice. Distribution of the fiber terminals in the area of hypothalamus is shown from bregma $-0.70 \mathrm{~mm}$ to bregma $-1.94 \mathrm{~mm}$. Scale bar, $250 \mu \mathrm{m}$. AHA anterior hypothalamic area, anterior part; AHC anterior hypothalamic area, central part; Arc arcuate hypothalamic nucleus; f fornix; LA lateroanterior hypothalamic nucleus; 3V 3rd ventricle; ic internal capsule; SI substantia innominate; AHP anterior hypothalamic area, posterior part; MTu medial tuberal nucleus; VMH ventromedial hypothalamic nucleus; MCLH magnocellular nucleus of the lateral hypothalamic; DM dorsomedial hypothalamic nucleus; PeF perifornical nucleus; PaLM paraventricular hypothalamic nucleus, lateral magnocellular part; SCh suprachiasmatic nucleus; MCPO magnocellular preoptic nucleus. b eGFP or hChR2-eGFP injected in the right alC and the terminals in LH were activated by light and immunostained with Fos (red) 30 min after light stimulation ( $n=5$ mice per group, Two-way ANOVA with Bonferroni post hoc analysis). Scale bar, $200 \mu \mathrm{m}$. c Light can evoke EPSCs (held at $-70 \mathrm{mV}$ ), but not IPSCs (held at $0 \mathrm{mV}$ ). $n=6$ cells. d Single light evoked EPSC, which can be blocked by CNQX. $n=5$ cells. e Diagram illustrating the injected virus (AAV-FLEX-eGFP-2A-TVA, AAV-FLEX-RVG, and $\Delta$ RVG-DsRed) (upper) and injection sites (lower) for rabies-based projection-specific monosynaptic tracing of inputs to LHVGluT2 neurons in Slc17a6-ires-Cre mice. Rabies are colocalized with CamKII in the right alC (upper). Neurons co-infected (starter cell) with helper AAV and rabies are shown in yellow. $\mathbf{f}$ Distribution of monosynaptic input (Rabies-DsRed neurons) from the right alC to the LHVGluT2. Scale bar, $100 \mu \mathrm{m}$ (Right upper). Scale bar, $25 \mu \mathrm{m}$ (Right lower). Source data are provided as a Source Data file.

Results from in situ hybridization of Slc17a6 mRNA showed that 94.5\% of CTB555-labeled neurons overlapped with Slc17a6 mRNA, indicating that VPMpc-to-aIC projecting neurons are primarily glutamatergic (Supplementary Fig. 10c).

The right aIC-to-LH projections mediate feeding suppression. To assess the functional contribution of the right aIC CamKII-to$\mathrm{LH}$ projection to feeding behavior, we introduced hChR2-eGFP into the caudal segment of the right aIC of Camk2a-Cre mice (Fig. 7a and Supplementary Fig. 11a). Photoactivation of the right aIC CamKII neuronal terminals in LH significantly reduced the amount of food intake, the number of approaches, and the percentage of time spent feeding in the fasted mice (two-tailed unpaired $t$ test, food intake, $t_{20}=2.445, P=0.0239$; number of approaches, $t_{20}=5.451, P<0.0001$; time feeding, $t_{20}=5.814, P<$ 0.0001 , Fig. $7 b, c)$ and also inhibited ongoing feeding when mice were in their home cages (two-tailed unpaired $t$ test, latency to stop, $t_{20}=15.33, \quad P<0.0001 ;$ feeding-inhibited stimulation, $t_{20}=14.31, P<0.0001$, Fig. $\left.7 \mathrm{~d}, \mathrm{e}\right)$. In addition, activation of the right aIC CamKII-to-LH pathway produced place avoidance (twotailed unpaired $t$ test, time in stimulation side, $t_{20}=3.493$, $P=0.0023$; velocity, 1 st light off step, $\mathrm{t}_{20}=0.5406, P=0.5948$, 1st light on step, $t_{20}=5.778, P<0.0001$, 2nd light off step, $\mathrm{t}_{20}=$ $0.7825, P=0.3862$, Fig. $7 \mathrm{f}, \mathrm{g})$, while it had no effects on drinking (two-tailed unpaired $t$ test, water intake, $t_{20}=0.523, P=0.6067$; number of licks, $t_{20}=0.5141, P=0.5942$; time drinking, $t_{20}=0.1318, P=0.8965$, Supplementary Fig. $\left.11 \mathrm{~b}\right)$ or mating behavior (two-tailed unpaired $t$ test, $t_{26}=0.1827, P=0.8565$, Supplementary Fig. 11c).

To confirm this observation, we used another labeling strategy. AAV8-hSyn-DIO-hChR2-eGFP virus was injected into the right aIC after a retrogradely transported virus canine adenovirus-2 encoding Cre-recombinase (Cav2-Cre) was injected into the $\mathrm{LH}$ (Supplementary Fig. 12a, b). This resulted in the specific hChR2 expression in the right aIC neurons that projected to $\mathrm{LH}$. Photoactivation of these neurons inhibited food consumption in $24 \mathrm{~h}$-fasted mice (Two-tailed unpaired $t$ test, food intake, 

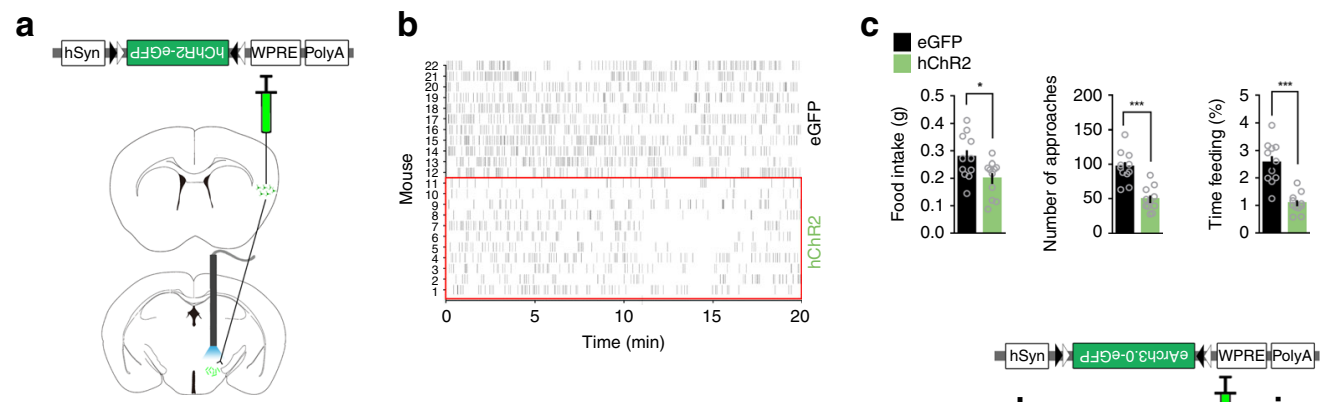

d
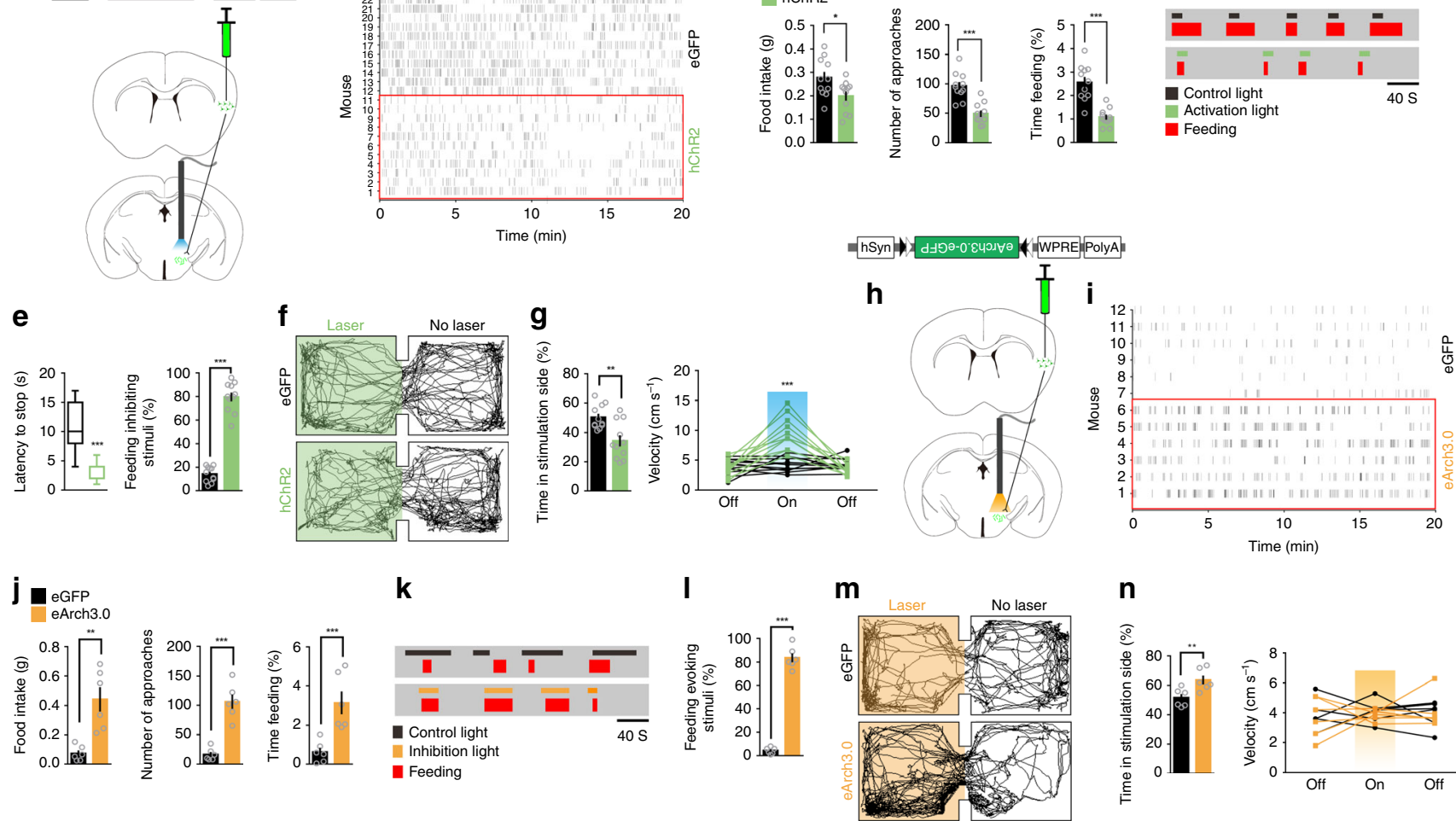

Fig. 7 Right alCCamKII-to-LH projections mediate feeding and avoidance behaviors. a Diagrams illustrating Camk2a-Cre mice injected with hChR2-eGFP in the right alC and photostimulated in the LH. b Raster plot of approaches to food in 24-h-fasted mice with the right alC CamKII-to-LH terminals being activated. c Activation of the right alCCamKII-to-LH terminals inhibits food intake in 24-h-fasted mice. Total food consumption, number of approaches and the percentage of feeding time were measured ( $n=11$ mice per group). $\mathbf{d}$ Raster plots showing feeding processes by one 24-h-fasted animal in its home cage. Activation light or control light was triggered after feeding began. e Latency to stop feeding (left) in response to light activation and the percentage of light stimuli that inhibits feeding ( $n=11$ mice per group). $\mathbf{f}$ Representative locomotor trace of a mouse with the right alCCamKII neurons expressing eGFP or hChR2 and the right $\mathrm{LH}$ receiving $20-\mathrm{Hz}$ photostimulation in the laser compartment. $\mathbf{g}$ Percentage of time spent in the photostimulation side (left) and locomotor activity in laser or no laser sides (right) ( $n=11$ mice per group). $\mathbf{h}$ Diagrams illustrating Camk2a-Cre mice with eArch3.0-eGFP injected in the right alC and photostimulation in the right LH. i Raster plot of feeding approach in fed mice with the right alC CamKII-to-LH terminals being photoinhibited. j Total food consumption, number of approaches to food, and the percentage of feeding time were measured ( $n=6$ mice per group). $\mathbf{k}$ Raster plots showing feeding processes by one fed animal in its home cage. Inhibition light or control light was triggered randomly. I The percentage of light stimulation that induces feeding ( $n=6$ mice per group). $\mathbf{m}$ Representative locomotor trace of a mouse with the right alC CamKII-to-LH terminals expressing eGFP or eArch3.0 and receiving photoinhibition in the laser compartment. $\mathbf{n}$ Percentage of time spent in the photoinhibition side and locomotor activity in laser or no laser sides ( $n=6$ mice per group). Two-tailed unpaired $t$ test, ${ }^{\star} P<0.05 ;{ }^{\star \star} P<0.01 ;{ }^{\star \star \star} P<0.005$. Data are presented as means \pm SEM. Source data are provided as a Source Data file.

$t_{8}=2.803, P=0.0230 ;$ number of approach, $t_{8}=3.153, P=$ 0.0275 ; time feeding, $t_{8}=4.786, P=0.0014$, Supplementary Fig. 12c) and induced place avoidance (Two-tailed unpaired $t$ test, time in stimulation side, $t_{8}=4.329, P=0.025$; velocity, 1 st light off step, $t_{8}=0.8011, P=0.44621$ st light on step, $t_{8}=8.526$, $P<0.0001$, 2nd light off step, $t_{8}=1.824, P=0.1056$, Supplementary Fig. 12d, e), while having no effects on drinking behavior (Two-tailed unpaired $t$ test, water intake, $t_{8}=0.7801, P=0.4578$; number of licks, $t_{8}=1.206, P=0.2623$; time drinking, $t_{8}=0.3376, P=0.7443$, Supplementary Fig. 12f), consistent with the aforementioned finding that the right aIC CamKII-to-LH pathway is sufficient to inhibit feeding and induce aversive effects.

In contrast, specifically silencing the right aIC CamKII-to-LH $^{\text {- }}$ projection terminals via introducing eArch3.0 in the right aIC CamKII neurons and stimulating projection terminals in the LH with 532-nm laser (Fig. 7h, Supplementary Fig. 13a) promoted food consumption in fed mice (Fig. 7i-l, Two-tailed unpaired $t$ test, for Supplementary Fig. $7 \mathrm{j}$, food intake, $t_{10}=4.341$, $P=0.0075$; number of approach, $t_{10}=5.593, P<0.0001$; time feeding, $t_{10}=4.082, P=0.0022$; for Supplementary Fig. 71 , $t_{10}=19.9, P<0.0001$ ), as well as place preference (Two-tailed unpaired $t$ test, time in stimulation side, $t_{20}=3.493, P=0.0023$; velocity, 1 st light off step, $t_{20}=0.5406, P=0.5948$, 1st light on step, $t_{20}=5.778, P<0.0001$, 2nd light off step, $t_{20}=0.7825$, $P=0.3862$, Fig. $7 \mathrm{~m}, \mathrm{n})$, while having no effects on drinking behavior (Two-tailed unpaired $t$ test, water intake, $t_{10}=1.253$, $P=0.2386$; number of licks, $t_{10}=1.949, \quad P=0.0798$; time drinking, $t_{10}=0.2572, P=0.8023$, Supplementary Fig. 13b). Taken together, these results indicate that the right aIC CamKII to-LH pathway mediates feeding suppression and place avoidance.

\section{Discussion}

In this study, we investigated how aversive stimuli alter insular activity and how direct manipulation of neuronal activity in the aIC affects aversive stimuli-induced feeding responses. Combining virus tracing, optogenetics, pharmacogenetics, photometry, and behavioral studies, we demonstrated that the caudal segment of the right aIC responds to diverse aversive stimuli in vivo and mediates anorexia via innervation of the LH. Therefore, we have identified a neural circuit from the cortex to the hypothalamus 
that interrupts the homeostatic system and mediates feeding responses under emergency conditions.

The insular cortex is known to process polymodal information about bodily states, including visceral ${ }^{34}$, gustatory ${ }^{35}$, somatosensory $^{36}$, and auditory modalities ${ }^{37}$. In this study, we identified a segment in the right aIC that gates feeding in response to aversive anorexigenic stimuli. Interestingly, when we compared it with the bitter center and sweet center identified by Zuker's group ${ }^{38}$, we found that this segment is just located between these two taste centers. These data demonstrate topographic segregation in the functional architecture of the insula ${ }^{39-41}$. It is possible that inputs carrying information from outside and inside the body project to topographically organized insular sensory regions. More tracing work is required to carefully analyze the connection between the insula and the thalamus or the PBN, such as the neuronal types, subregion, and left-right difference that serves sensory function.

The LH is a critical neuroanatomical region that is tied to consummatory and motivational behaviors. vGAT and vGluT2expressing LH neurons produce a bidirectional output signal, which is directly or indirectly conveyed to the ventral tegmental area (VTA) dopamine neurons to homeostatically invigorate behavioral output. Inhibitory GABAergic subcortical fibers that innervate $\mathrm{LH}$ may originate from the lateral septum ${ }^{42}$ and much of the basal forebrain and the extended amygdala, including the nucleus accumbens shell ${ }^{43,44}$, the bed nucleus of the stria terminalis (BNST)/preoptic area ${ }^{45}$, the ventral pallidum ${ }^{46}$ and nucleus basalis/substantia innominate ${ }^{47}$. However, the source of excitatory inputs to the LH is still unknown. In this study, we showed that the glutamatergic input from the aIC to the LH inhibits food intake, consistent with the previous finding that vGluT2expressing LH neurons produce an output signal to inhibit feeding and induce place avoidance. Therefore, we propose a model that environmental representations, such as aversive visceral stimuli, are likely encoded in the right insular cortex, which in turn convey representational information to the $\mathrm{LH}$ neuronal circuits to regulate feeding and rewarding. Previous work has shown that the LH may exert its effect on feeding directly via projecting to the $\mathrm{PBN}^{48,49}$ to suppress feeding behavior or indirectly to the lateral habenula $(\mathrm{LHb})^{50}$ or $\mathrm{VTA}^{51}$ by affecting the rewarding aspect of food consumption. More work is required to identify downstream brain regions that elicit the feeding suppression function of the aIC-to-LH circuit.

Craig 6 has proposed laterality differences in interoceptive perception related to emotional processing for the insular cortex in humans. In this study, we found that the right but not the left aIC in mice is critical in sensing aversive visceral stimuli and controlling feeding behavior. Moreover, inactivation of the right but not the left aIC reverses chemotherapy-induced weight loss. Consistently, one previous study in rats has also reported lateralized CREB activation in conditioned taste aversion with the right IC being more obvious than the left $\mathrm{IC}^{52}$. These findings suggest that hemispheric functional lateralization of aIC is not unique to humans but may be present in rodents as well. Furthermore, our findings are reminiscent of the right hemispheric dominance in negative emotional processing and expression ${ }^{53,54}$ and of the right aIC hemispheric dominance in interoceptive attention in humans ${ }^{15,55-57}$. Recently, more and more evidence in rodents indicates left-right asymmetry for cognitive and emotional faculties. Several brain regions have been reported to be lateralized for corresponding behavioral functions, such as anterior cingulate cortex (ACC) for observational fear ${ }^{58,59}$, mPFC for anxiety ${ }^{60}$, amygdala for pain and fear ${ }^{61-64}$, hypothalamus for energy metabolism 65,66 , and hippocampus for learning and spatial navigation ${ }^{67-69}$, respectively. However, further evidence is required to understand brain left-right asymmetries at microscopic levels involving molecules, synapses, neurons, and neuronal circuits. Our findings here may provide an entry point for addressing basic neuronal underpinnings of insular functions and malfunctions in an accessible animal model.

To conclude, in this study, we have identified a pathway from the right insular cortex to the right $\mathrm{LH}$ that controls the intake of food. Additionally, to avoid potential confounding effects caused by lateralization, care should be taken in future behavioral studies when examining the role of a circuit.

\section{Methods}

Animals. Camk2a-Cre mice (B6.Cg-Tg(Camk2a-cre $)^{T 29-1 S t l} / \mathrm{J}$, strain 005359, RRID: IMSR_JAX:005359) and Slc17a6-ires-Cre mice (B6J.129S6(FVB)-Slc17a6 ${ }^{\text {tm2 (cre) }}$ Lowl/MwarJ, strain 028863, RRID:IMSR_JAX:028863) were obtained from the Jackson Laboratory. The male mice were used for behavioral test, and both the male and female mice were used for tracing image. Camk2a-Cre, Slc17a6-ires-Cre and C57BL/6J mice at 2-4 months of age were housed on a 12-h light/dark cycle under standard conditions in the animal facility with food and water ad libitum unless otherwise noted.

All animal care and experimental procedures complied with all relevant ethical regulations, were strictly conducted in accordance with the Guidelines for the Care and Use of Laboratory Animals of Zhejiang University and were approved by the Institutional Animal Care and Use Committee at Zhejiang University.

Regents. CNO, LiCl, LPS, Cisplatin, CNQX, and DAPI dye were obtained from Sigma Aldrich. Rat Ghrelin and mouse cholecysokinin (CCK) were obtained from Tocris Bioscience. CTB, Alexa Fluor ${ }^{\mathrm{TM}} 555$ were obtained from Thermo Fisher.

Viral constructs. The following AAV viruses were provide by Shanghai Taitool Bioscience Co., Ltd: AAV9-hSyn-DIO-hM3Dq-mCherry, AAV9-hSyn-DIOhM4Di-mCherry, AAV8-hSyn-DIO-mCherry, AAV8-hSyn-DIO-eGFP, AAV9CamKIIa-mCherry, AAV8-hSyn-DIO-hChR2(H134R)-eGFP, AAV8-CamKIIahChR2(H134R)-eGFP, AAV9-hSyn-DIO-eArch3.0-eGFP, AAV9-mCamKIIaGCaMP6f, AAV9-hSyn-DIO-mCherry, and Cav2-CMV-Cre. AAV9-CAG-DIOTVA-EGFP, AAV9-CAG-DIO-RG and EnvA-pseudotyped, glycoprotein (RG)deleted and DsRed-expressing rabies virus (RV-EvnA-DsRed, RV) were provided by Wuhan BrainVTA Co., Ltd.

Stereotaxic surgery. 2-4 months old mice were deeply anesthetized with 5\% isoflurane (vol per vol) in oxygen and then kept with $1.5 \%$ isoflurane. Surgery was performed with a stereotaxic frame (Stoelting). For caudal segment of aIC injection, $150 \mathrm{nl}$ of virus was injected unilaterally on site $(+0.5 \mathrm{~mm}$ antero-posterior (AP); $\pm 3.85 \mathrm{~mm}$ medio-lateral $(\mathrm{ML}) ;-2.82 \mathrm{~mm}$ dorso-ventral (DV) relative to Bregma). For right posterior insular cortex (pIC), virus was injected unilaterally on site $(-1.10 \mathrm{~mm}$ AP; $-3.90 \mathrm{~mm} \mathrm{ML} ;-3.25 \mathrm{~mm}$ DV relative to Bregma). For rostral segment of the right aIC, virus was injected unilaterally on site (+1.6 mm AP; -3.1 $\mathrm{mm} \mathrm{ML} ;-1.8 \mathrm{~mm}$ DV relative to Bregma). Virus was injected into each location at $0.03 \mu \mathrm{l} / \mathrm{min}$. The syringe was not removed until 15-20 $\mathrm{min}$ after the end of infusion to allow diffusion of the virus. After injection, mice were single housed and allowed 2-4 weeks for viral expression and recovery from surgery.

For soma photostimulation or photoinhibition experiments, recombinant AAVs expressing hChR2 (H134R), eArch3.0, or eGFP were unilaterally injected into the aIC of Camk2a-Cre mice or wild-type mice. Three weeks after surgery, a fibreoptic cannula with $0.22 \mathrm{NA}$ or $0.37 \mathrm{NA}$ was implanted above the injected aIC $(-2.67 \mathrm{~mm} \mathrm{DV})$ and mice were allowed another 1 week for recovery from surgery before behavioral test.

For axon terminal photostimulation experiments, recombinant AAVs expressing hChR2(H134R), eArch3.0, or eGFP were unilaterally injected into the aIC of Camk2a-Cre mice. Three weeks after surgery, a fibreoptic cannula with 0.22 NA was implanted above the $\mathrm{LH}(-1.28 \mathrm{~mm} \mathrm{AP}, \pm 1.23 \mathrm{~mm} \mathrm{ML},-5.28 \mathrm{~mm} \mathrm{DV})$. Mice were allowed another 1 week for recovery from surgery before behavioral test.

For chemogenetic stimulation or inhibition, recombinant AAVs expressing hM3Dq, hM4Di, or mCherry were injected into the caudal segment of the aIC of Camk2a-Cre mice unilaterally. Four weeks later, the mice were received an i.p. injection of CNO $(1 \mathrm{mg} / \mathrm{kg})$ or the equivalent volume of saline and were allowed to recover in their home cage for $30 \mathrm{~min}$ before behavioral test. The mice with virus distributed mainly from Bregma $+1.34 \mathrm{~mm}$ to Bregma $+0.02 \mathrm{~mm}$ were used to analyze the related behaviors.

For photometry experiments, recombinant AAVs expressing mCamKIIaGCaMP6f were injected unilaterally into the aIC of wild-type mice. Two weeks later, a fibreoptic cannula with 0.37 NA was implanted above the injected aIC $(-2.67 \mathrm{~mm} \mathrm{DV})$. Mice were allowed 1 week for recovery from surgery before behavioral test.

For projection-mapping experiments, recombinant AAVs expressing GFP were injected unilaterally into the aIC of Camk2a-Cre mice. Eight weeks later, mice were sacrificed, fixed, and sections were collected for imaging. 
For monosynaptic-tracing experiments, Slc17a6-ires-Cre mice were microinjected unilaterally in the LH with $150 \mathrm{nl}$ viral cocktail (1:1) with AAVCAG-DIO-TVA-EGFP to allow the initial infection of LH starter neurons. AAVCAG-DIO-RG coding for the rabies virus envelope glycoprotein was also injected into the LH at the same time to allow the trans-synaptic spread of virus. Three weeks later, $200 \mathrm{nl}$ of the modified rabies virus was microinjected into the same location. Another one week later, mice were sacrificed and fixed. Brain sections were collected for imaging.

Immunohistochemistry. Mice were sacrificed and perfused with 4\% PFA in PBS. Brains were fixed overnight in PFA, cryoprotected in $30 \%$ sucrose (wt per vol) at $4{ }^{\circ}$ $\mathrm{C}$ for $24 \mathrm{~h}$, and then coronally sectioned at $40 \mu \mathrm{m}$ on a cryostat (Leica CM1900). Brain sections were blocked with $10 \%$ NGS and $0.3 \%$ Triton-X in PBS for $1 \mathrm{~h}$ at room temperature, and then incubated with primary antibody (rabbit anti-Fos, SYSY system, 226 003, 1:10,000, RRID:AB_2231974; mouse anti-CamKII, Abcam, ab22609, 1:500, RRID:AB 447192; mouse anti-GAD67, Millipore, MAB5406, 1:1,000, RRID:AB_2278725) at $4{ }^{\circ} \mathrm{C}$ for $48 \mathrm{~h}$. Sections were then washed with PBS for three times and incubated with secondary antibody (Alexa Fluor 488 goat antirabbit, Life Technologies, a11008, 1:2000, RRID:AB_143165; Alexa Fluor 555 donkey anti-rabbit, Life Technologies, a10040, 1:2000, RRID:AB_2534016; Alexa Fluor 488 goat anti-mouse, Life Technologies, a11029, 1:2000, RRID:AB 138404) for $2 \mathrm{~h}$ at room temperature. Sections were mounted and imaged with an Olympus VS120 microscope. For detection of Fos in ChR2 or GFP control mice, animals received 10-min of $20-\mathrm{Hz}, \sim 5 \mathrm{~mW}$ photostimulation $(488 \mathrm{~nm})$ and were perfused 30 min later. For detection of Fos in mice with different aversive stimuli, mice were perfused $1.5 \mathrm{~h}$ after injection (i.p.) with different regents or electric foot shock unless otherwise mentioned.

RNAscope in situ hybridization. Animals were anesthetized and perfused with DEPC-PBS followed by ice-cold 4\% PFA in DEPC-PBS. Brains were dissected and post-fixed over night at $4{ }^{\circ} \mathrm{C}$ and dehydrated with $30 \%$ sucrose in DPEC-PBS Afterward, brains were sectioned at $20 \mu \mathrm{m}$ thickness and mounted directly onto glass slides. RNAscope assay were performed according to RNAscope ${ }^{\circledast}$ Multiplex Fluorescent Reagent Kit v2 User Manual (ACD Bio). Probe against Slc17a6 (vGluT2) mRNA was obtained from ACD Bio (Mm-Slc17a6-319171). Images were captured with an Olympus VS120 microscope.

Acute brain-slice preparation and electrophysiology. The mouse was deeply anesthetized with diethyl ether and the brain was rapidly removed and placed in ice-cold, high-sucrose cutting solution containing (in $\mathrm{mM}$ ): 194 sucrose, $30 \mathrm{NaCl}$, $26 \mathrm{NaHCO}_{3}, 10$ glucose, $4.5 \mathrm{KCl}, 1.2 \mathrm{NaH}_{2} \mathrm{PO}_{4}, 7 \mathrm{MgSO}_{4}, 0.2 \mathrm{CaCl}_{2}$, and $2 \mathrm{MgCl}_{2}$. Slices were cut on a Leica vibratome (VT1200S) in the high-sucrose cutting solution and immediately transferred to an incubation chamber with artificial cerebrospinal fluid (ACSF) containing (in mM) $119 \mathrm{NaCl}, 26.2 \mathrm{NaHCO}_{3}, 11$ glucose, $2.5 \mathrm{KCl}, 1 \mathrm{NaH}_{2} \mathrm{PO}_{4}, 1.3 \mathrm{MgCl}_{2}$, and $2.5 \mathrm{CaCl}_{2}$. The slices were allowed to recover at $34^{\circ} \mathrm{C}$ for $30 \mathrm{~min}$ before being allowed to equilibrate at room temperature for another hour. During recordings, the slices were placed in a recording chamber constantly perfused with warmed ACSF $\left(28-30^{\circ} \mathrm{C}\right)$ and gassed continuously with $95 \% \mathrm{O}_{2}$ and $5 \% \mathrm{CO}_{2}$. For whole-cell voltage-clamp recording, the recording pipettes (3-5 M $\Omega$ ) were filled with a Cs-based low $\mathrm{Cl}^{-}$internal solution containing (in mM): $135 \mathrm{CsMeSO}_{3}, 10$ HEPES, 1 EGTA, 3.3 QX-314, 4Mg-ATP, $0.3 \mathrm{Na}$-GTP, 8 $\mathrm{Na} 2$-phosphocreatine, $290 \mathrm{mOsm} \mathrm{kg}^{-1}$, adjusted to $\mathrm{pH} 7.3$ with $\mathrm{CsOH}$. For wholecell current-clamp recording, the recording pipettes were filled with (in $\mathrm{mM}$ ) 110 K-gluconate, $40 \mathrm{KCl}, 10 \mathrm{HEPES}, 2 \mathrm{Mg}$-ATP, $0.5 \mathrm{Na}_{2}$-GTP, and 0.2 EGTA 290 mOsm $\mathrm{kg}^{-1}$, adjusted to $\mathrm{pH} 7.3$ with KOH. Data were digitized at $10 \mathrm{kHz}$, collected with a MultiClamp 700B amplifier, and analyzed by pClamp10 software (Molecular Devices, Sunnyvale, USA). Membrane potential was held at $-70 \mathrm{mV}$ to record AMPAR-mediated current and at $0 \mathrm{mV}$ to record $\mathrm{GABA}_{\mathrm{A}}$ receptor-mediated IPSC The following drugs were used diluted in ACSF as indicated: $100 \mu \mathrm{M}$ Picrotoxin (PTX) and $20 \mu \mathrm{M}$ CNQX. For confirmation of the efficiency of chemogenetic activation (hM3Dq) or silencing (hM4Di) of synaptic transmission, $5 \mu \mathrm{M}$ of $\mathrm{CNO}$ diluted in ACSF was used. For ChR2 validation and circuit mapping, a blue lightemitting diode (NWEDOON) controlled by digital commands from the $1440 \mathrm{~A}$ was connected to the epifluorescence port of an Olympus BX51 microscope to deliver photostimulation. To record light-evoked EPSCs, $1-2 \mathrm{~ms}$ at $\lambda=473 \mathrm{~nm}$ and $1-10 \mathrm{~mW} / \mathrm{mm}^{2}$ blue light was delivered through the objective to illuminate the entire field of view. For optogenetic silencing validation, cells were activated using currents ranging from 0 to $45 \mathrm{pA}(\Delta I=5 \mathrm{pA})$ for $3 \mathrm{~s}$ in duration injected under current clamp mode and photosilenced using a 532-nm LED light source (NEWDOON) sending constant light at $10 \mathrm{~mW} / \mathrm{mm}^{2}$.

In vivo fiber photometry. AAV-mCamKII $\alpha$-GCaMP6f was injected unilaterally into the aIC. Two weeks later, optic-fiber cannulas (fiber: core $=230 \mu \mathrm{m}, \mathrm{NA}=$ 0.37 , NEWDOON) were implanted $0.15 \mathrm{~mm}$ above to the injection side. Behavioral test started one week later to allow for recovery from surgery. To record fluorescence signals, laser beam from a 488-nm laser (OBIS 488LS; Coherent) was reflected by a dichroic mirror (MD498; Thorlabs), focused by a $\times 10$ objective lens $(\mathrm{NA}=0.3$; Olympus) and then coupled to an optical commutator (Doric Lenses). To record fluorescence signals, laser beam from a 488-nm laser (OBIS 488LS;
Coherent) was reflected by a dichroic mirror (MD498; Thorlabs), focused by a $\times 10$ objective lens $(\mathrm{NA}=0.3$; Olympus) and then coupled to an optical commutator (Doric Lenses). An optical fiber ( $230 \mu \mathrm{m}$ O.D., NA $=0.37,2-\mathrm{m}$ long) guided the light between the commutator and the implanted optical fiber. The laser power was adjusted at the tip of optical fiber to the low level of $0.01-0.02 \mathrm{~mW}$ to minimize bleaching. The GCaMP fluorescence was bandpass filtered (MF525-39, Thorlabs) and collected by a photomultiplier tube (R3896, Hamamatsu). An amplifier (C7319, Hamamatsu) was used to convert the photomultiplier tube current output to voltage signals, which was further filtered through a low-pass filter $(40 \mathrm{~Hz}$ cutoff; Brownlee 440). The analog voltage signals were digitalized at $500 \mathrm{~Hz}$ and recorded by a Power 1401 digitizer.

For chemogenetic assay, mice were acclimated to the behavioral chamber (30 $\mathrm{cm} \times 15 \mathrm{~cm} \times 55 \mathrm{~cm})$ for $30 \mathrm{~min}$, and then injected intraperitoneally with $\mathrm{LiCl}(150$ $\mathrm{mg} / \mathrm{kg}$ ) or Cisplatin $(4 \mathrm{mg} / \mathrm{kg})$. Calcium signals were recorded for another $30 \mathrm{~min}$ Photometry setting, including laser power and time constant, were the same for every mouse and every recording session. Photometry data were subjected to minimal processing consisting of only within-trial fluorescence normalization.

In vivo photostimulation. For all optical activation studies, mice received blue light laser stimulation ( $473 \mathrm{~nm}$, NEWDOON) of $\sim 5 \mathrm{~mW}$ with a 10 ms pulse width For optical inhibition studies, mice received constant green light stimulation (532 $\mathrm{nm}, \mathrm{NEWDOON}$ ) of $\sim 10 \mathrm{~mW}$. The lasers were triggered and pulses were controlled with Intelligent light system software.

Animal behaviors. All mice used in behavioral assays were allowed to recover from surgery of AAV injection and Ferrule fibers implantation for at least 4 weeks.

Feeding behavior for optogenetic assay. Before the test, mice were transferred into an empty testing cage in the behavioral testing room to habituate for at least 1 $\mathrm{h}$. For $24 \mathrm{~h}$-fasted feeding test, mice were food-deprived the day before test, with water provided ad libitum. The mice were then briefly anesthetized with isoflurane and coupled with optic fibers. $20 \mathrm{~min}$ after recovery, mice were introduced into the test cage with a regular food pellet (Chow), or anorexigenic agents (CCK, $\mathrm{LiCl}$, LPS) allowed for feeding for $20 \mathrm{~min}$. The weight of the food pellet, including the food debris left in the cage floor after test, was measured to calculate the food intake. For the fed feeding test, mice were not food deprived before testing and allowed to feed for $20 \mathrm{~min}$. For optogenetic experiments, the light was started just before the mice were introduced into the testing cage or home cage. The feeding behavior was videotaped and manually analyzed.

Feeding behavior for pharmacogenetic assay. For chemogenetic stimulation studies, mice were injected with recombination AAV expressing hM3Dq 4 weeks before experiment. The mice were then given ad libitum with Chow 1 week prior to, during, and after the behavioral test. CNO $(1 \mathrm{mg} / \mathrm{kg})$ was injected twice a day for 7 days intraperitoneally. The body weight of mice was measured every day between 1 p.m. and 5 p.m.

For chemogenetic inhibition assay, mice were injected unilaterally into the aIC with recombination AAV expressing hM4Di 4 weeks before experiment. Mice were given ad libitum access to food prior to, during, and after the assay. Cisplatin (4 $\mathrm{mg} / \mathrm{kg}$ ) was administered every 2 days intraperitoneally for 2 weeks. CNO (1 mg/ $\mathrm{kg}$ ) was injected intraperitoneally twice a day for 7 days after Cisplatin administered for 1 week. The body weight of mice was measured every day between 1 p.m. and 5 p.m.

Taste sensitivity for optogenetic assay. Taste sensitivity was measured with free-moving mice in behavioral chamber. Prior to behavior test, mice were water deprived for $24 \mathrm{~h}$. Before test mice were briefly anesthetized with isoflurane and coupled with optic fibers. 20 min after recovery, mice were introduced into the test cage with water, $0.01 \mathrm{mM}$ quinine, $0.1 \mathrm{mM}$ quinine, and $1 \mathrm{mM}$ quinine for $20-\mathrm{min}$ drinking test. The total numbers of lick were videotaped and manually analyzed.

Water consumption assay. Mice were water-restricted for $24 \mathrm{~h}$ in their home cage, acclimated to the behavioral chamber for $15 \mathrm{~min}$, and then provided with access to water for $20 \mathrm{~min}$. The weight of the water, total number of licks, and percent of drinking time after test was measured to calculate.

Elevated plus-maze test. The elevated plus-maze consisted of a plus-shaped platform with four intersecting arms: two opposing open arms and two closed arms. Animals were placed in the center of the apparatus facing a closed arm and allowed to freely explore the maze for $5 \mathrm{~min}$. The parameters such as time and distance traveled in the open arm were analyzed with the smart3.0 (Panlab) software. The area was cleaned with $75 \%$ ethanol between tests.

Open field test. OFT was performed in an open field arena $(50 \mathrm{~cm}$ long, $50 \mathrm{~cm}$ wide, and $60 \mathrm{~cm}$ high). Experiments were conducted under low light conditions in order to minimize anxiety effects. Mice were allowed to freely explore for $5 \mathrm{~min}$. 
The parameter center entries time was analyzed with the smart 3.0 software. The area was cleaned with $75 \%$ ethanol between tests.

Real-time place preference assay. Place preference training was performed in a custom-made two-compartment conditioned place preference (CPP) apparatus $(30 \mathrm{~cm} \times 25 \mathrm{~cm} \times 20 \mathrm{~cm})$. After connecting with optical fiber, mice infected with AAV-hChR2 or AAV-eGFP were placed in the CPP training apparatus for $20 \mathrm{~min}$ to assess their baseline place preference. During the test, we assigned the counterbalanced side of the chamber as the stimulation side, and placed the mice in the non-stimulated side to start the experiment. When the mouse crossed to the stimulated side of the chamber, it triggered $5 \mathrm{~Hz}$ laser stimulation $(473-\mathrm{nm}, 10 \mathrm{~ms}$ pulses) until the mouse crossed back to the non-stimulated side. Avoidance score was calculated by subtracting the time spent in stimulation side during baseline (without light) from the time spent in stimulation side during the test (with light).

Measurement of heart rate. Mice were anesthetized with $5 \%$ isoflurane (vol per vol) in oxygen and then kept with $1.5 \%$ isoflurane. Heart rate data were analyzed after band pass filtering $(10-200 \mathrm{~Hz}$, Butterworth three-order filter). Heart beats per minute (BPM) were determined by custom-written Matlab program ${ }^{70,71}$. At least, three calculations from each animal were averaged over a period of $3 \mathrm{~s}$ recording or longer.

Data analysis. For photometry assay, all data were analyzed with custom-written Matlab program. For photometry data, all responses were normalized to baseline using the function: $\Delta F / F=\left(F-F_{0}\right) / F_{0}$, in which $F_{0}$ is the median fluorescence of the baseline. The baseline period for full experiments was $30 \mathrm{~min}$ before time zero. To analyze the responses during feeding, the control time window was set $2 \mathrm{~s}$ before food touch onset to minimize potential false-positive effects. $\Delta F / F$ values are presented as average plots with a gray area indicating the \pm SEM. Locomotor activity for mouse was analyzed using a video tracking software (Smart v3.0). We then averaged the value $>10$ trials for food consumption. To analyze the responses during aversive stimulation, the control time window was set $60 \mathrm{~s}$ before IP injection and averaged the value for 3-4 mouse for aversive stimulation test

For rabies tracing quantification, the rabies-labeled inputs into brain regions were quantified and normalized to the sum of starter cells in LH. Three brains were used for each experiment.

For axon projection quantification, images were obtained and quantified with three junction sections for each region and were averaged to obtain the final value. Three brains were used for each experiment.

Quantification of Fos staining was performed on every 6th slice in the LH from Bregma -0.7 to $-1.92 \mathrm{~mm}$ (six sections per mice) and on every third slice in the following area: PBN from Bregma -5.02 to $-5.40 \mathrm{~mm}$ (three sections per mice), NTS from Bregma -6.96 to $-7.48 \mathrm{~mm}$ (five sections per mice), BLA from Bregma -0.58 to $-1.34 \mathrm{~mm}$ (six sections per mice), mPFC from Bregma +1.98 to +1.54 $\mathrm{mm}$ (five sections per mice), and VPMpc from Bregma -1.82 to $-1.94 \mathrm{~mm}$ (two sections per mice). Quantification of Fos staining in the aIC in Fig. 1a-d and Supplementary Fig. 3d, or quantification of colocalization of Fos with CamKII or GAD67 in Fig. 1e, $f$ was performed on every 6th slice in the aIC from Bregma +1.18 to $+0.02 \mathrm{~mm}$ (four sections per mice). Quantification of Fos staining in the whole IC in Supplementary Fig. $2 \mathrm{a}-\mathrm{f}$ was performed on every third slice from Bregma +2.46 to $-1.06 \mathrm{~mm}$ ( 28 sections per mice). Quantification of Fos staining in the aIC in Supplementary Fig. $2 \mathrm{~g}$, $\mathrm{h}$ was performed on every third slice in the IC from Bregma +1.18 to $+0.5 \mathrm{~mm}$ (seven sections per mice). All images were subsequently overlaid with the corresponding atlas section to anatomically define the regions of interest. Positive cells lying on the boundary were excluded. A cell was considered positive only if it displayed an intensity value above the intensity threshold of the background. Six brains were used for each experiment. Quantification was performed using the cell counter tool in ImageJ.

Statistical analysis. Statistical analyses were performed using Matlab or Prism 7 (GraphPad). Throughout the paper, the level of significance is indicated as ${ }^{*} P<$ $0.05,{ }^{* *} P<0.01,{ }^{* * *} P<0.005$. No statistical methods were used to predetermine the sample sizes. Pairwise comparisons were calculated with unpaired two-tailed $t$ tests, and multiple group data comparisons were calculated with one-way or two-way ANOVA with Bonferroni post hoc test. Normality was assessed with Shapiro-Wilk tests. If normality tests failed, Mann-Whitney or Wilcoxon rank-sum tests were used. Cell count, anatomical, and behavioral analysis were fully and/or partially conducted by experimenters blind to experimental conditions.

Reporting summary. Further information on research design is available in the Nature Research Reporting Summary linked to this article.

\section{Data availability}

The source data underlying Figs. 1b, d, f, 2e, g, h, k, 3c, e, g, 4c, e, 5h, i, 6b, 7c, e, g, j, l, and n and Supplementary Figs. 1b, 2b-f, h, 3b-d, 4c, e, 5b, d, f, i, k, m, 6b, d, 7b, c, e, g, 8b, c, e, g, 9c, 10b, d, 11b, c, 12c, e, f and 13b are provided as a Source Data file. All relevant data are available from the corresponding authors upon reasonable request.
Received: 19 January 2019; Accepted: 16 December 2019; Published online: 31 January 2020

\section{References}

1. Sternson, S. M. \& Eiselt, A. K. Three pillars for the neural control of appetite. Annu. Rev. Physiol. 79, 401-423 (2017).

2. Sternson, S. M. Hypothalamic survival circuits: blueprints for purposive behaviors. Neuron 77, 810-824 (2013).

3. Cowley, M. A. et al. The distribution and mechanism of action of ghrelin in the CNS demonstrates a novel hypothalamic circuit regulating energy homeostasis. Neuron 37, 649-661 (2003).

4. Waterson, M. J. \& Horvath, T. L. Neuronal regulation of energy homeostasis: beyond the hypothalamus and feeding. Cell Metab. 22, 962-970 (2015).

5. Dietrich, M. O., Zimmer, M. R., Bober, J. \& Horvath, T. L. Hypothalamic Agrp neurons drive stereotypic behaviors beyond feeding. Cell 160, 1222-1232 (2015).

6. Craig, A. D. How do you feel-now? The anterior insula and human awareness. Nat. Rev. Neurosci. 10, 59-70 (2009).

7. Craig, A. D. Emotional moments across time: a possible neural basis for time perception in the anterior insula. Philos. Trans. R. Soc. Lond. B 364, 1933-1942 (2009).

8. Shura, R. D., Hurley, R. A. \& Taber, K. H. Insular cortex: structural and functional neuroanatomy. J. Neuropsychiatry Clin. Neurosci. 26, 276-282 (2014).

9. Gogolla, N. The insular cortex. Curr. Biol. 27, R580-R586 (2017).

10. Bliss, T. V., Collingridge, G. L., Kaang, B. K. \& Zhuo, M. Synaptic plasticity in the anterior cingulate cortex in acute and chronic pain. Nat. Rev. Neurosci. 17, 485-496 (2016).

11. Berman, D. E. \& Dudai, Y. Memory extinction, learning anew, and learning the new: dissociations in the molecular machinery of learning in cortex. Science 291, 2417-2419 (2001).

12. Shema, R., Hazvi, S., Sacktor, T. C. \& Dudai, Y. Boundary conditions for the maintenance of memory by PKMzeta in neocortex. Learn. Mem. 16, 122-128 (2009).

13. Inberg, S. et al. Fluid consumption and taste novelty determines transcription temporal dynamics in the gustatory cortex. Mol. Brain 9, 13 (2016).

14. Blonde, G. D., Bales, M. B. \& Spector, A. C. Extensive lesions in rat insular cortex significantly disrupt taste sensitivity to $\mathrm{NaCl}$ and $\mathrm{KCl}$ and slow salt discrimination learning. PLoS ONE 10, e0117515 (2015).

15. Critchley, H. D. Electrodermal responses: what happens in the brain. Neuroscientist 8, 132-142 (2002).

16. Schienle, A., Schafer, A., Hermann, A. \& Vaitl, D. Binge-eating disorder: reward sensitivity and brain activation to images of food. Biol. Psychiatry 65 , 654-661 (2009).

17. Frank, G. K., Shott, M. E., Riederer, J. \& Pryor, T. L. Altered structural and effective connectivity in anorexia and bulimia nervosa in circuits that regulate energy and reward homeostasis. Transl. Psychiatry 6, e932 (2016).

18. Nunn, K., Frampton, I., Fuglset, T. S., Torzsok-Sonnevend, M. \& Lask, B. Anorexia nervosa and the insula. Med. Hypotheses 76, 353-357 (2011).

19. Holsen, L. M. et al. Food motivation circuitry hypoactivation related to hedonic and nonhedonic aspects of hunger and satiety in women with active anorexia nervosa and weight-restored women with anorexia nervosa. $J$. Psychiatry Neurosci. 37, 322-332 (2012).

20. Livneh, Y. et al. Homeostatic circuits selectively gate food cue responses in insular cortex. Nature 546, 611-616 (2017).

21. Contreras, M., Ceric, F. \& Torrealba, F. Inactivation of the interoceptive insula disrupts drug craving and malaise induced by lithium. Science 318, 655-658 (2007).

22. Cai, H., Haubensak, W., Anthony, T. E. \& Anderson, D. J. Central amygdala PKC-delta $(+)$ neurons mediate the influence of multiple anorexigenic signals. Nat. Neurosci. 17, 1240-1248 (2014).

23. Qiu, S. et al. An increase in synaptic NMDA receptors in the insular cortex contributes to neuropathic pain. Sci. Signal. 6, ra34 (2013).

24. Adaikkan, C. \& Rosenblum, K. A molecular mechanism underlying gustatory memory trace for an association in the insular cortex. Elife 4, e07582 (2015).

25. Oppenheimer, S. M., Gelb, A., Girvin, J. P. \& Hachinski, V. C. Cardiovascular effects of human insular cortex stimulation. Neurology 42, 1727-1732 (1992).

26. Critchley, H. D. \& Harrison, N. A. Visceral influences on brain and behavior Neuron 77, 624-638 (2013).

27. Zito, K. A., Bechara, A., Greenwood, C. \& van der Kooy, D. The dopamine innervation of the visceral cortex mediates the aversive effects of opiates. Pharm. Biochem. Behav. 30, 693-699 (1988). 
28. Bermudez-Rattoni, F. \& McGaugh, J. L. Insular cortex and amygdala lesions differentially affect acquisition on inhibitory avoidance and conditioned taste aversion. Brain Res. 549, 165-170 (1991).

29. Ramirez-Amaya, V., Alvarez-Borda, B. \& Bermudez-Rattoni, F. Differential effects of NMDA-induced lesions into the insular cortex and amygdala on the acquisition and evocation of conditioned immunosuppression. Brain Behav. Immun. 12, 149-160 (1998).

30. Naqvi, N. H., Rudrauf, D., Damasio, H. \& Bechara, A. Damage to the insula disrupts addiction to cigarette smoking. Science 315, 531-534 (2007).

31. Baldo, B. A., Spencer, R. C., Sadeghian, K. \& Mena, J. D. GABA-mediated inactivation of medial prefrontal and agranular insular cortex in the rat: contrasting effects on hunger- and palatability-driven feeding. Neuropsychopharmacology 41, 960-970 (2016).

32. Alhadeff, A. L. et al. Excitatory hindbrain-forebrain communication is required for cisplatin-induced anorexia and weight loss. J. Neurosci. 37, 362-370 (2017).

33. Stuber, G. D. \& Wise, R. A. Lateral hypothalamic circuits for feeding and reward. Nat. Neurosci. 19, 198-205 (2016).

34. Cechetto, D. F. Central representation of visceral function. Fed. Proc. 46, 17-23 (1987)

35. Yamamoto, T., Yuyama, N., Kato, T. \& Kawamura, Y. Gustatory responses of cortical neurons in rats. I. Response characteristics. J. Neurophysiol. 51, 616-635 (1984).

36. Rodgers, K. M., Benison, A. M., Klein, A. \& Barth, D. S. Auditory, somatosensory, and multisensory insular cortex in the rat. Cereb. Cortex 18, 2941-2951 (2008).

37. Sawatari, H. et al. Identification and characterization of an insular auditory field in mice. Eur. J. Neurosci. 34, 1944-1952 (2011).

38. Peng, Y. et al. Sweet and bitter taste in the brain of awake behaving animals. Nature 527, 512-515 (2015).

39. Morris, R., Frey, S., Kasambira, T. \& Petrides, M. Ibotenic acid lesions of the basolateral, but not the central, amygdala interfere with conditioned taste aversion: evidence from a combined behavioral and anatomical tract-tracing investigation. Behav. Neurosci. 113, 291-302 (1999).

40. Reilly, S. \& Bornovalova, M. A. Conditioned taste aversion and amygdala lesions in the rat: a critical review. Neurosci. Biobehav. Rev. 29, 1067-1088 (2005).

41. Roman, C., Nebieridze, N., Sastre, A. \& Reilly, S. Effects of lesions of the bed nucleus of the stria terminalis, lateral hypothalamus, or insular cortex on conditioned taste aversion and conditioned odor aversion. Behav. Neurosci. 120, 1257-1267 (2006).

42. Anthony, T. E. et al. Control of stress-induced persistent anxiety by an extraamygdala septohypothalamic circuit. Cell 156, 522-536 (2014).

43. Heimer, L., Zahm, D. S., Churchill, L., Kalivas, P. W. \& Wohltmann, C. Specificity in the projection patterns of accumbal core and shell in the rat. Neuroscience 41, 89-125 (1991).

44. Zahm, D. S. An electron microscopic morphometric comparison of tyrosine hydroxylase immunoreactive innervation in the neostriatum and the nucleus accumbens core and shell. Brain Res. 575, 341-346 (1992).

45. Jennings, J. H., Rizzi, G., Stamatakis, A. M., Ung, R. L. \& Stuber, G. D. The inhibitory circuit architecture of the lateral hypothalamus orchestrates feeding. Science 341, 1517-1521 (2013).

46. Root, D. H., Melendez, R. I., Zaborszky, L. \& Napier, T. C. The ventral pallidum: subregion-specific functional anatomy and roles in motivated behaviors. Prog. Neurobiol. 130, 29-70 (2015).

47. Grove, E. A. Neural associations of the substantia innominata in the rat: afferent connections. J. Comp. Neurol. 277, 315-346 (1988).

48. Norgren, R. Gustatory responses in the hypothalamus. Brain Res. 21, 63-77 (1970).

49. Li, C. S., Cho, Y. K. \& Smith, D. V. Modulation of parabrachial taste neurons by electrical and chemical stimulation of the lateral hypothalamus and amygdala. J. Neurophysiol. 93, 1183-1196 (2005).

50. Matsumoto, M. \& Hikosaka, O. Lateral habenula as a source of negative reward signals in dopamine neurons. Nature 447, 1111-1115 (2007).

51. Mangieri, L. R. et al. A neural basis for antagonistic control of feeding and compulsive behaviors. Nat. Commun. 9, 52 (2018).

52. Duerden, E. G., Arsalidou, M., Lee, M. \& Taylor, M. J. Lateralization of affective processing in the insula. Neuroimage 78, 159-175 (2013).

53. Adolphs, R., Damasio, H., Tranel, D. \& Damasio, A. R. Cortical systems for the recognition of emotion in facial expressions. J. Neurosci. 16, 7678-7687 (1996).

54. Ahern, G. L. \& Schwartz, G. E. Differential lateralization for positive and negative emotion in the human brain: EEG spectral analysis. Neuropsychologia 23, 745-755 (1985)

55. Craig, A. D. How do you feel? Interoception: the sense of the physiological condition of the body. Nat. Rev. Neurosci. 3, 655-666 (2002).
56. Critchley, H. D., Mathias, C. J. \& Dolan, R. J. Fear conditioning in humans: the influence of awareness and autonomic arousal on functional neuroanatomy. Neuron 33, 653-663 (2002).

57. Critchley, H. D., Wiens, S., Rotshtein, P., Ohman, A. \& Dolan, R. J. Neural systems supporting interoceptive awareness. Nat. Neurosci. 7, 189-195 (2004).

58. Keum, S. et al. A missense variant at the Nrxn3 locus enhances empathy fear in the mouse. Neuron 98, 588-601 e585 (2018).

59. Kim, S., Matyas, F., Lee, S., Acsady, L. \& Shin, H. S. Lateralization of observational fear learning at the cortical but not thalamic level in mice. Proc. Natl Acad. Sci. USA 109, 15497-15501 (2012).

60. Costa, N. S., Vicente, M. A., Cipriano, A. C., Miguel, T. T. \& Nunes-de-Souza, R. L. Functional lateralization of the medial prefrontal cortex in the modulation of anxiety in mice: Left or right? Neuropharmacology 108, 82-90 (2016).

61. Baker, K. B. \& Kim, J. J. Amygdalar lateralization in fear conditioning: evidence for greater involvement of the right amygdala. Behav. Neurosci. 118, 15-23 (2004).

62. Scicli, A. P., Petrovich, G. D., Swanson, L. W. \& Thompson, R. F. Contextual fear conditioning is associated with lateralized expression of the immediate early gene c-fos in the central and basolateral amygdalar nuclei. Behav. Neurosci. 118, 5-14 (2004).

63. Kolber, B. J. et al. Activation of metabotropic glutamate receptor 5 in the amygdala modulates pain-like behavior. J. Neurosci. 30, 8203-8213 (2010).

64. Sadler, K. E. et al. Divergent functions of the left and right central amygdala in visceral nociception. Pain 158, 747-759 (2017).

65. Toth, I. et al. Hypothalamic sidedness in mitochondrial metabolism: new perspectives. Reprod. Sci. 21, 1492-1498 (2014).

66. Toth, I. et al. Estrogen- and satiety state-dependent metabolic lateralization in the hypothalamus of female rats. PLoS ONE 10, e0137462 (2015).

67. Igloi, K., Doeller, C. F., Berthoz, A., Rondi-Reig, L. \& Burgess, N. Lateralized human hippocampal activity predicts navigation based on sequence or place memory. Proc. Natl Acad. Sci. USA 107, 14466-14471 (2010).

68. Denenberg, V. H., Garbanati, J., Sherman, D. A., Yutzey, D. A. \& Kaplan, R. Infantile stimulation induces brain lateralization in rats. Science 201, 1150-1152 (1978).

69. Klur, S. et al. Hippocampal-dependent spatial memory functions might be lateralized in rats: an approach combining gene expression profiling and reversible inactivation. Hippocampus 19, 800-816 (2009).

70. Zehendner, C. M., Luhmann, H. J. \& Yang, J. W. A simple and novel method to monitor breathing and heart rate in awake and urethane-anesthetized newborn rodents. PLoS ONE 8, e62628 (2013).

71. Salay, L. D., Ishiko, N. \& Huberman, A. D. A midline thalamic circuit determines reactions to visual threat. Nature 557, 183-189 (2018).

\section{Acknowledgements}

This work was supported by the National Natural Science Foundation of China (91732102, 81471125, and 81671049 to S.Q., 31900722 to Y.W.), the Zhejiang Science Fund for Distinguished Young Scholars (LR16C090001 to S.Q.), the Fundamental Research Funds for the Central Universities of China (2019XZZX001-01-14 to S.Q.) the Chinese Ministry of Education Project 111 Program (B13026 to S.Q.), and Certificate of China Postdoctoral Science Foundation Grant (2018M630665 to Y.W.). CIHR operating grant (MOP-124807) and project grant (PJT-148648), Azrieli Neurodevelopmental Research Program and Brain Canada (M.Z.). We thank Dr. Shumin Duan (Zhejiang University) for providing us with Slc17a6-ires-Cre mice and Dr. Jianhong Luo (Zhejiang University) for providing us with Camk2a-Cre mice. We thank Yudong Zhou (Zhejiang University), Hailan Hu (Zhejiang University), and Tatiana Korotkova (Max Planck Institute for Metabolism Research) for valuable discussion. We also thank the technical support by the Core Facilities, Zhejiang University School of Medicine.

\section{Author contributions}

Y.W., C.W.C., and M.C. performed the viral injection, animal behavior, and immunostaining experiments; collected, analyzed, and interpreted data; and participated in writing the paper. X.Y.L. performed the electrophysiological experiments; collected, analyzed, and interpreted data; and participated in writing the paper. K.Q. and H.T.W performed immunohistochemical staining. Y.W. and L.F.J. performed the calciumimaging experiments; and collected, analyzed, and interpreted data. L.Y. and M.Z. contributed intellectually and revised the manuscript. S.Q. was responsible for the overall supervision of the study; designed the experiments; analyzed and interpreted data; and revised the paper.

\section{Competing interests}

The authors declare no competing interests. 


\section{Additional information}

Supplementary information is available for this paper at https://doi.org/10.1038/s41467020-14281-5.

Correspondence and requests for materials should be addressed to S.Q.

Peer review information Nature Communications thanks Kobi Rosenblum, Richard Palmiter and Zhiping Pang for their contribution to the peer review of this work. Peer reviewer reports are available.

Reprints and permission information is available at http://www.nature.com/reprints

Publisher's note Springer Nature remains neutral with regard to jurisdictional claims in published maps and institutional affiliations. (c) (i) Open Access This article is licensed under a Creative Commons Attribution 4.0 International License, which permits use, sharing, adaptation, distribution and reproduction in any medium or format, as long as you give appropriate credit to the original author(s) and the source, provide a link to the Creative Commons license, and indicate if changes were made. The images or other third party material in this article are included in the article's Creative Commons license, unless indicated otherwise in a credit line to the material. If material is not included in the article's Creative Commons license and your intended use is not permitted by statutory regulation or exceeds the permitted use, you will need to obtain permission directly from the copyright holder. To view a copy of this license, visit http://creativecommons.org/ licenses/by/4.0/.

(C) The Author(s) 2020 